\title{
mRNA-1273 protects against SARS-CoV-2 beta infection in nonhuman primates
}

\author{
Kizzmekia S. Corbett ${ }^{1,9}$, Anne P. Werner ${ }^{1}{ }^{1}$, Sarah O' Connell', Matthew Gagne', Lilin Lai², \\ Juan I. Moliva', Barbara Flynn', Angela Choi ${ }^{3}$, Matthew Koch ${ }^{3}$, Kathryn E. Foulds ${ }^{1}$, Shayne F. Andrew', \\ Dillon R. Flebbe1, Evan Lamb', Saule T. Nurmukhambetova', Samantha J. Provost', Kevin W. Bock ${ }^{4}{ }^{4}$, \\ Mahnaz Minai ${ }^{4}$, Bianca M. Nagata4, Alex Van Ry ${ }^{5}{ }^{5}$, Zackery Flinchbaugh ${ }^{5}$, Timothy S. Johnston (1), \\ Elham Bayat Mokhtari', Prakriti Mudvari', Amy R. Henry', Farida Laboune', Becky Chang ${ }^{5}$, \\ Maciel Porto5, Jaclyn Wear5, Gabriela S. Alvarado', Seyhan Boyoglu-Barnum', John-Paul M. Todd', \\ Bridget Bart ${ }^{5}$, Anthony Cook ${ }^{5}$, Alan Dodson ${ }^{5}$, Laurent Pessaint ${ }^{5}$, Katelyn Steingrebe ${ }^{5}$, Sayda Elbashir ${ }^{3}$, \\ Manjari Sriparna ${ }^{1}{ }^{1}$, Andrew Pekosz ${ }^{6}$, Hanne Andersen ${ }^{5}{ }^{5}$, Kai Wu ${ }^{3}$, Darin K. Edwards ${ }^{3}$, Swagata Kar ${ }^{5}$,

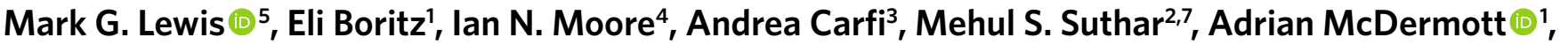 \\ Mario Roederer ${ }^{1}$, Martha C. Nason ${ }^{8}$, Nancy J. Sullivan', Daniel C. Douek ${ }^{1}$, Barney S. Graham ${ }^{1 凶}$ and \\ Robert A. Seder (1) 1 屁
}

B.1.351 is the severe acute respiratory syndrome coronavirus 2 (SARS-CoV-2) variant most resistant to antibody neutralization. We demonstrate how the dose and number of immunizations influence protection. Nonhuman primates received two doses of 30 or $100 \mu \mathrm{g}$ of Moderna's mRNA-1273 vaccine, a single immunization of $30 \mu \mathrm{g}$, or no vaccine. Two doses of $100 \mu \mathrm{g}$ of mRNA1273 induced 50\% inhibitory reciprocal serum dilution neutralizing antibody titers against live SARS-CoV-2 p.Asp614Gly and B.1.351 of 3,300 and 240, respectively. Higher neutralizing responses against B.1.617.2 were also observed after two doses compared to a single dose. After challenge with B.1.351, there was $\sim 4-$ to $5-\log _{10}$ reduction of viral subgenomic RNA and low to undetectable replication in bronchoalveolar lavages in the two-dose vaccine groups, with a $1-\log _{10}$ reduction in nasal swabs in the 100- $\mu$ g group. These data establish that a two-dose regimen of mRNA-1273 will be critical for providing upper and lower airway protection against major variants of concern.

T he emergence of SARS-CoV-2 variants of concern (VOCs) that show reduced neutralization by convalescent or vaccine-induced immune serum from the Wuhan-1 strain ${ }^{1-3}$ has created uncertainty about the efficacy of current SARS-CoV-2 vaccines against VOC infection. So far, the most concerning variants contain combinations of mutations and deletions in the spike (S) protein receptor-binding domain (RBD) and N-terminal domain (NTD). Acquisition of amino acid substitutions in the $S$ RBD, namely p.Lys417Asn, p.Glu484Lys and p.Asn501Tyr, and in the NTD, such as p.Leu18Phe, p.Asp80Ala, p.Asp215Gly and $\Delta 242-244$, is associated with increased transmissibility and reduction in neutralization sensitivity ${ }^{4-11}$. Variants containing these substitutions, originally isolated in the United Kingdom (UK; B.1.1.7, Alpha), Republic of South Africa (B.1.351, Beta), Brazil (P.1 lineage, Gamma), New York (B.1.526) and California (B.1.427/B.1.429), have shown varying reductions in neutralization by convalescent and vaccine sera and are resistant to some monoclonal antibodies $^{10,12-18}$. Moreover, the B.1.617.2 (Delta) variant is now the most prevalent variant circulating globally and shows some resistance to neutralization by sera from vaccinated individuals ${ }^{19,20}$.

Among these variants, B.1.351 contains the most mutations in the RBD and NTD subdomains ${ }^{21}$ and has been shown to have the largest fold reduction in neutralizability by potent RBD-specific monoclonal antibodies, including LY-CoV555 (ref. ${ }^{10}$ ), and convalescent serum from individuals infected with ancestral SARS-CoV-2 strains $^{6,22,23}$. Additionally, it was reported that sera from mRNA-1273-immunized human and nonhuman primates (NHPs) showed the greatest reduction of neutralization against B.1.351 compared to B.1.1.7, P.1, B.1.427/B.1.429 and B.1.1.7+Glu484Lys variants $^{4-11,24-26}$. In UK-based or US-based clinical studies, NVX-CoV2373 (Novavax), AZD1222 (University of Oxford/ AstraZeneca) and Ad26.COV2.S (Janssen/Johnson \& Johnson)

\footnotetext{
'Vaccine Research Center; National Institute of Allergy and Infectious Diseases, National Institutes of Health, Bethesda, MD, USA. ${ }^{2}$ Center for Childhood Infections and Vaccines of Children's Healthcare of Atlanta, Department of Pediatrics, Department of Microbiology and Immunology, Emory Vaccine Center, Emory University, Atlanta, GA, USA. ${ }^{3}$ Moderna, Cambridge, MA, USA. ${ }^{4}$ Infectious Disease Pathogenesis Section, National Institute of Allergy and Infectious Diseases, National Institutes of Health, Bethesda, MD, USA. ${ }^{5}$ Bioqual, Rockville, MD, USA. ${ }^{6}$ Department of Microbiology and Immunology, Johns Hopkins University, Bloomberg School of Public Health, Baltimore, MD, USA. ${ }^{7}$ Department of Microbiology and Immunology, Emory University, Atlanta, GA, USA. ${ }^{8}$ Biostatistics Research Branch, Division of Clinical Research, National Institute of Allergy and Infectious Diseases, National Institutes of Health, Baltimore, MD, USA. ${ }^{9}$ Present address: Department of Immunology and Infectious Diseases, Harvard T.H. Chan School of Public Health, Boston, MA, USA. 凶e-mail: bgraham@nih.gov; rseder@mail.nih.gov
} 
vaccines show between $\sim 70$ and $90 \%$ protection against the circulating p.Asp614Gly or B.1.1.7 variants ${ }^{7,27-29}$, and vaccine efficacy against mild symptomatic coronavirus disease 2019 (COVID-19) caused by B.1.351 was up to $60 \%$ for Ad26.CoV2 (ref. ${ }^{29}$ ) and NVX-CoV2373 (ref.$^{30}$ ) and $\sim 10 \%$ for AZD122 (refs. ${ }^{7,27-29}$ ). A recent report showed that BNT162b2, Pfizer's mRNA vaccine, conferred $~ 75 \%$ protection against confirmed B.1.351 infection in Qatar ${ }^{31}$. While immunological assessments for all vaccine trials are underway and correlates of protection are not yet determined, these data highlight the potential impact that reduced neutralization capacity to B.1.351 may have on protection against mild symptomatic COVID-19 across various platforms. Although comparable to BNT162b2 in other settings, human efficacy trials with mRNA-1273 have not been conducted in regions where B.1.351 circulates as a dominant variant ${ }^{32}$.

Vaccine development for COVID-19 has benefited from clinically translatable data from the NHP model ${ }^{33-39}$. As there have been no published studies on vaccine protection in NHPs challenged with the B.1.351 variant, we evaluated the impact of the dose and number of immunizations with mRNA-1273 on immunogenicity and protection against B.1.351 challenge in NHPs. Here, we show a correlation between high mRNA-1273-induced antibody responses and decreased viral replication following B.1.351 challenge to establish that a two-dose regimen of mRNA-1273 will be critical for providing upper and lower airway protection against major VOCs.

\section{Results}

Antibody responses following mRNA-1273 vaccination. In previous studies, vaccination of NHPs with $10-100 \mu \mathrm{g}$ of mRNA-1273 at weeks 0 and 4 conferred rapid and complete control of detectable viral replication in both the upper and lower airways following SARS-CoV-2 USA/Washington-1 (WA-1) challenge ${ }^{33,38}$. In the current study, to assess the influence of dose and number of immunizations on immunogenicity and protection against B.1.351, NHPs were immunized with 30 or $100 \mu \mathrm{g}$ in the standard 0-week and 4 -week vaccine regimen $(\times 2)$ or a single dose $(\times 1)$ of $30 \mu \mathrm{g}$ (Extended Data Fig. 1).

We first performed a temporal analysis of serum neutralizing antibody responses after single immunization or prime and boost with mRNA-1273. Consistent with a previous study ${ }^{38}$, there was a $~ 100$-fold increase in p.Asp614Gly-specific lentiviral pseudovirus neutralizing antibodies following a boost with 30 or $100 \mu \mathrm{g}$ of mRNA-1273 (Extended Data Fig. 2a). All 8 of the $100 \mu \mathrm{g} \times 2$ immunized NHPs and 7 of 8 receiving $30 \mu \mathrm{g} \times 2$ had $>10^{3}$ reciprocal $50 \%$ inhibitory dose $\left(\mathrm{ID}_{50}\right)$ titers $7-8$ weeks after boost, and 13 of 16 animals from both two-dose regimens had detectable neutralizing activity against B.1.351 (Extended Data Fig. 2b). By contrast, only 6 of 8 NHPs that received a single dose of $30 \mu \mathrm{g}$ had detectable neutralizing responses against p.Asp614Gly at that same time point, and none $(0 / 8)$ had detectable neutralizing antibodies against B.1.351 (Extended Data Fig. 2a,b).

Focusing immune analysis at the time of SARS-CoV-2 B.1.351 challenge, $\sim 8$ weeks after boost or 12 weeks after the single immunization, S-specific binding and neutralizing antibody responses were assessed. Using a 10-plex MULTI-ARRAY ELISA, we assessed WA-1 (Fig. 1a,e) and B.1.351 (Fig. 1d,h) S-specific and RBD-specific antibody binding responses, which represent the vaccine and challenge strains, respectively. B.1.351 S-specific (Fig. 1a,d) and RBD-specific (Fig. 1e,h) antibody responses were reduced by less than twofold to threefold for each vaccine group as compared to WA-1 S. There was a vaccine dose-dependent increase in S-specific and RBD-specific antibody responses against WA-1 (Fig. 1a,e), B.1.351 (Fig. 1d,h), B.1.1.7 (Fig. 1b,f) and P.1 (Fig. 1c,g).

In vitro neutralizing activity was next determined using two pseudovirus neutralization assays and a live-virus assay. Neutralizing titers against B.1.351 were compared to p.Asp614Gly, the benchmark strain. Using a p.Asp614Gly lentiviral-based pseudovirus neutralization assay, the reciprocal $\mathrm{ID}_{50}$ geometric mean titer (GMT) was 3,600 following two doses of $100 \mu \mathrm{g}$ (Fig. 1i and Extended Data Fig. 2a). Consistent with the eightfold reduction reported by us and others using human vaccine or convalescent serum ${ }^{3,20,26,40}$, the reciprocal $\mathrm{ID}_{50}$ GMT against B.1.351 was $\sim 450$ (Fig. 1i and Extended Data Fig. 2b). Notably, in NHPs that received a single $30-\mu$ dose of mRNA, the reciprocal ID $_{50}$ GMT against p.Asp614Gly was $\sim 130$, but there were no detectable neutralizing antibodies against B.1.351 (Fig. 1i). We observed similar outcomes comparing D614G to B.1.351 using vesicular stomatitis virus (VSV)-based pseudovirus (Fig. 1j) and live-virus (Fig. 1k) neutralization assays. To extend the analysis, there was little change in neutralization in any vaccine group comparing p.Asp614Gly to B.1.1.7 (Fig. 11) but the reduction in neutralization against the P.1 variant (Fig. $1 \mathrm{~m}$ ) was similar to that observed with B.1.351 (Fig. 1i). Last, there was an average threefold reduction in GMT (half-maximal effective concentration $\left.\left(\mathrm{EC}_{50}\right)\right)$ in serum neutralizing activity against B.1.617.2 compared to p.Asp614Gly in animals that received two doses of 30 or $100 \mu \mathrm{g}$ of mRNA-1273 and undetectable responses in the 30- $\mu$ g single-dose group. Nevertheless, reciprocal $\mathrm{ID}_{50}$ neutralizing responses were $\sim 400$ and $\sim 750$ in the 30- $\mu$ g and $100-\mu \mathrm{g}$ two-dose groups, respectively. Antibody binding and neutralization responses were highly correlated with one another (Extended Data Fig. 3).

To extend the antibody analyses to the mucosal sites of infection, S-specific and RBD-specific IgG and IgA in bronchoalveolar lavage (BAL) and nasal wash samples were assessed at $\sim 3$ weeks after boost or 7 weeks after the single $30-\mu \mathrm{g}$ immunization. Consistent with systemic humoral responses, there was a dose-dependent increase in BAL and nasal wash WA-1 or B.1.351 S-specific IgG and IgA (Fig. 2a-h), BAL WA-1 (Fig. 2a) or B.1.351 (Fig. 2b). S-specific IgA responses in nasal wash samples trended higher only in the two-dose vaccine groups compared to control NHPs (Fig. $2 \mathrm{~g}, \mathrm{~h}$ ). RBD-specific antibody responses followed similar trends to S-specific responses (Fig. 2i-p). Overall, mRNA-1273 vaccination elicits WA-1 and B.1.351 S-specific IgG and IgA antibodies in serum and lower and upper airways, as previously shown ${ }^{38}$.

T cell responses following mRNA-1273 vaccination. mRNA1273 induces type 1 helper $\mathrm{T}\left(\mathrm{T}_{\mathrm{H}} 1\right)$ cells, $\mathrm{CD}^{+}$follicular helper $\mathrm{T}\left(\mathrm{T}_{\mathrm{FH}}\right)$ responses and CD8 ${ }^{+} \mathrm{T}$ cells in NHPs and humans ${ }^{33,41,42}$. Consistent with these data, $\mathrm{S}$-specific $\mathrm{T}_{\mathrm{H}} 1$ responses were induced in a dose-dependent manner with higher responses in the 100- $\mu \mathrm{g}$ group (Fig. 3a). There were low to undetectable type 2 helper T $\left(\mathrm{T}_{\mathrm{H}} 2\right)$ cell responses in all vaccine groups (Fig. 3b). There was

Fig. 1 | Serum antibody responses following mRNA-1273 immunization. a-n, Twenty-four rhesus macaques were immunized with mRNA-1273 (30 $\mu$ g, one dose (light blue) or two doses (dark blue); or $100 \mu \mathrm{g}$, two doses (red)), according to Extended Data Fig. 1. Eight aged-matched naive NHPs (gray) were used as controls. Sera collected at week 12, immediately before challenge, were assessed for SARS-CoV-2 USA/WA-1 (a and e), B.1.1.7 (b and f), P.1 (c and $\mathbf{g}$ ) and B.1.351 (d and h) S-specific (a-d) and RBD-specific (e-h) IgG by MULTI-ARRAY ELISA. Lentiviral-based pseudovirus neutralization (i and I-n) was conducted on SARS-CoV-2 p.Asp614Gly and B.1.351 (i), B.1.1.7 (I), P.1 (m) and B.1.617.2 (n). p.Asp614Gly and B.1.351 VSV-based pseudovirus neutralization (j) and focus-reduction live-virus neutralization (k) were also assessed. Data represent one independent experiment. a-h, Circles represent individual NHPs. Boxes and horizontal bars denote the interquartile range (IQR) and medians, respectively; whisker end points are equal to the maximum and minimum values. i-n, Gray lines represent individual NHPs, and colored lines represent GMTs. Dotted lines indicate neutralization assay limits of detection. Symbols represent individual NHPs and may overlap for equal values. Data represent one independent experiment. AUC, area under the curve. 
also dose dependence in the frequency of $\mathrm{S}$-specific $\mathrm{T}_{\mathrm{FH}}$ responses expressing the surface marker CD40L (Fig. 3c) or the canonical cytokine interleukin (IL)-21 (Fig. 3d), which are critical for improving antibody responses. S-specific $\mathrm{CD}^{+} \mathrm{T}$ cell responses were observed in five of eight NHPs that received two doses of $100 \mu \mathrm{g}$ mRNA-1273 (Fig. 3e). These data show that mRNA-1273 induces $\mathrm{T}_{\mathrm{H}} 1$-skewed and $\mathrm{T}_{\mathrm{FH}^{-}}$-skewed $\mathrm{CD} 4$ responses and $\mathrm{CD}^{+}$ $\mathrm{T}$ cells at the highest dose.

Protection against SARS-CoV-2 replication in the airways. An extensive analysis was performed to characterize the nucleotide sequence and in vivo pathogenicity. Deep sequencing was
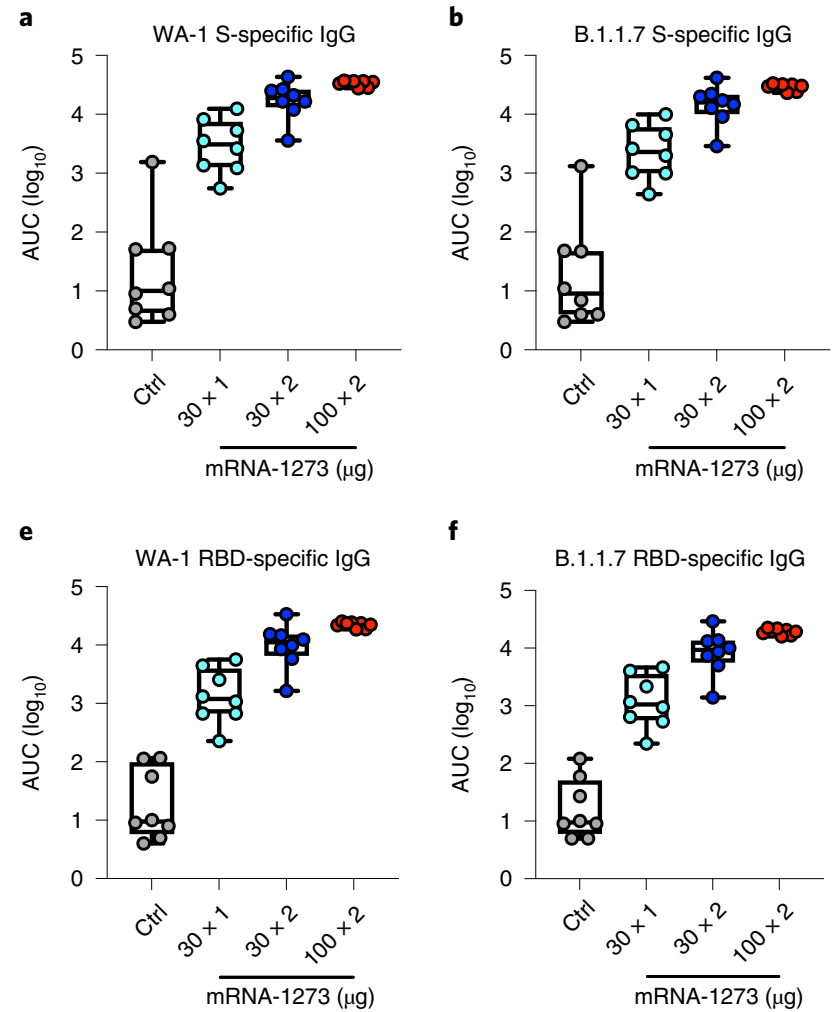

g
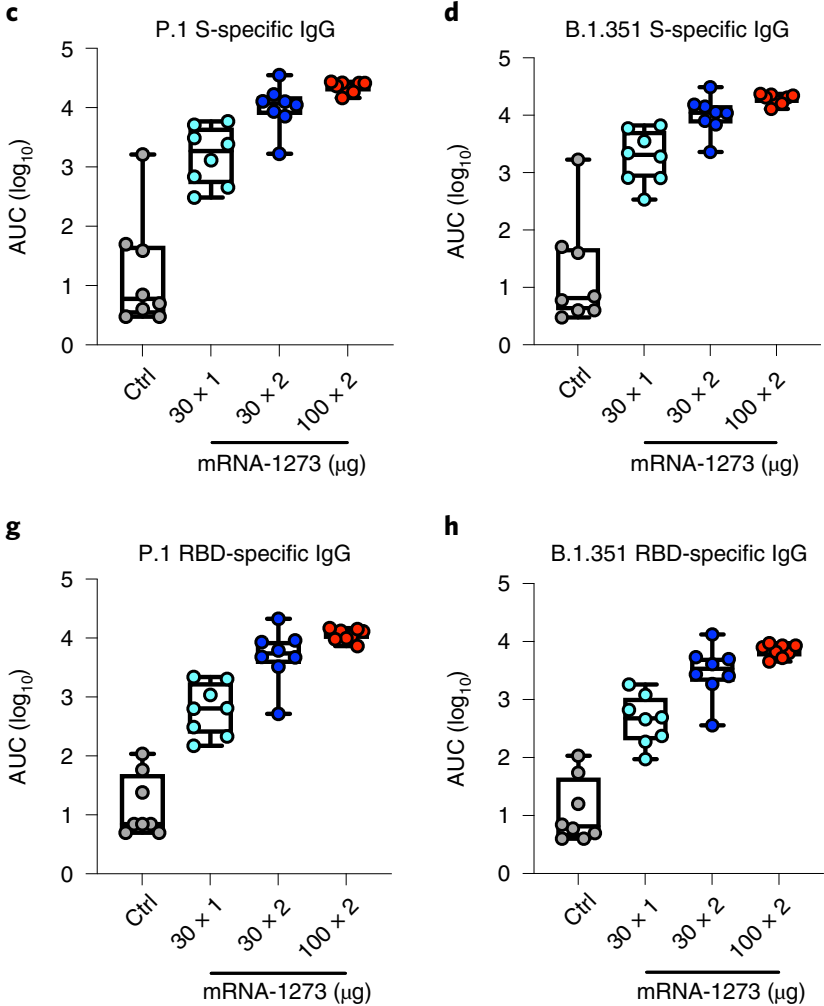

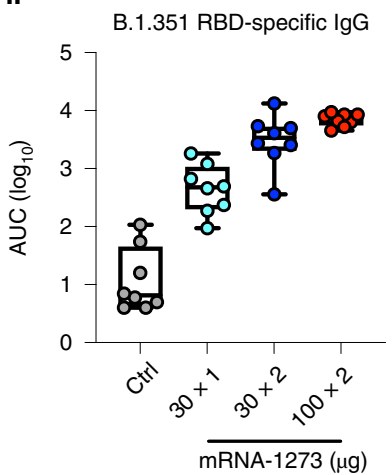

i

Lentiviral pseudovirus neutralization p.Asp614Gly vs. B.1.351

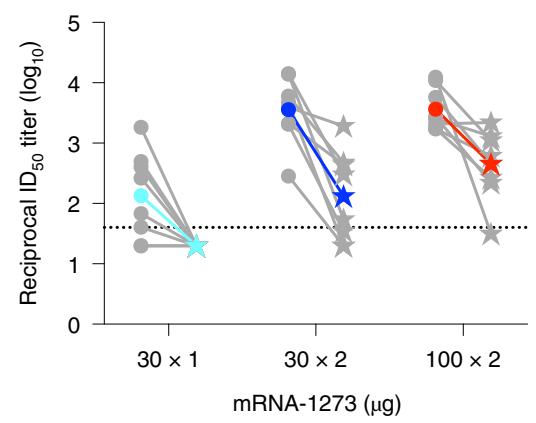

I

Lentiviral pseudovirus neutralization p.Asp614Gly vs. B.1.1.7

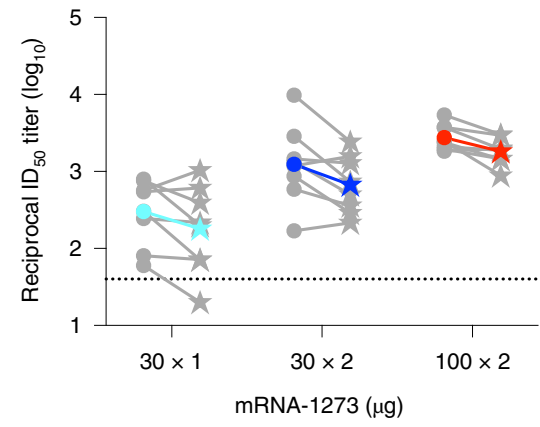

j

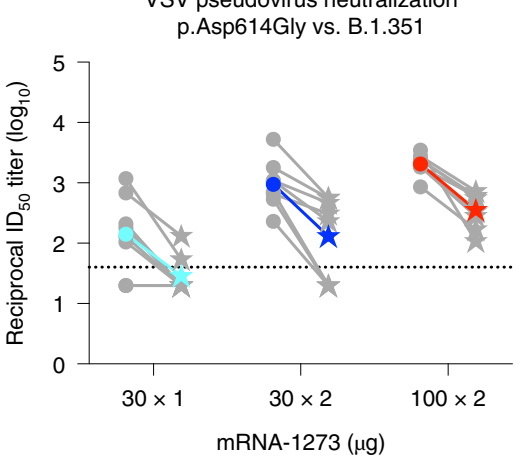

$\mathbf{m}$

Lentiviral pseudovirus neutralization p.Asp614Gly vs. P.1

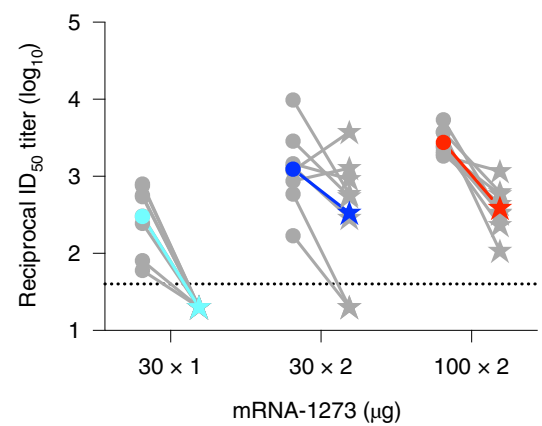

$\mathbf{k}$
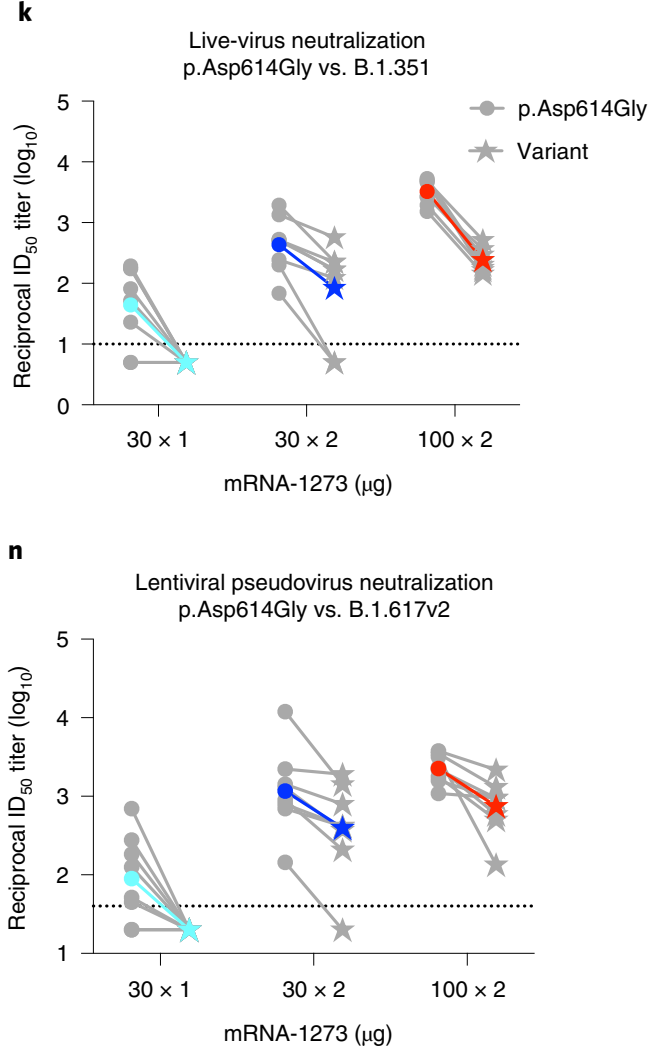
a

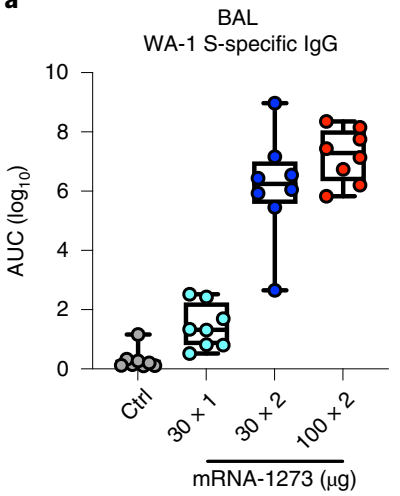

e
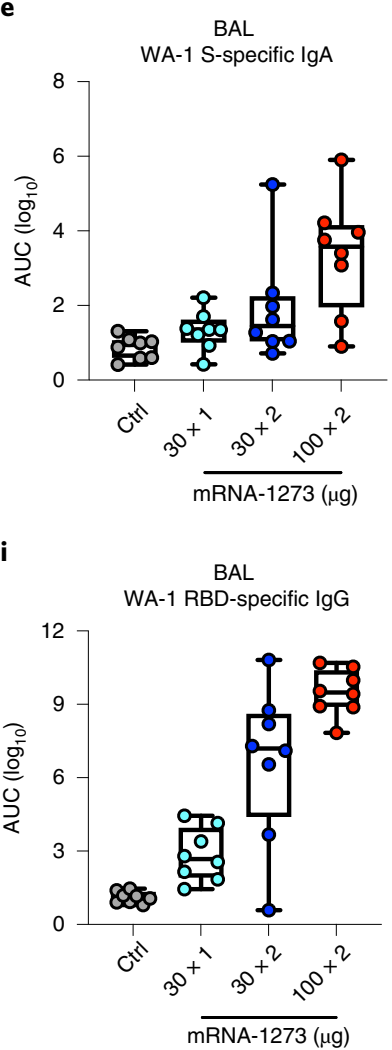

m

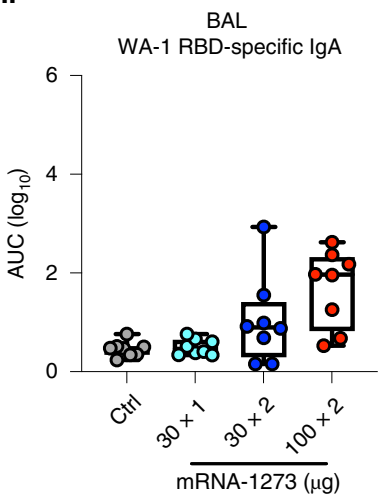

b

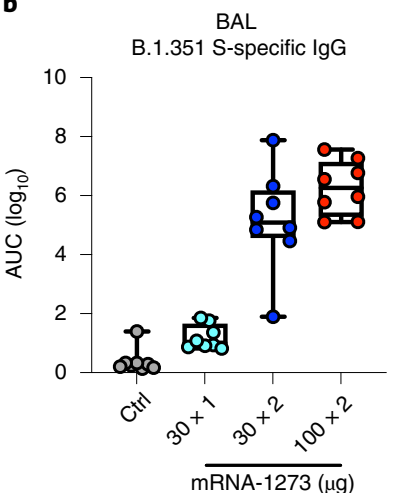

f

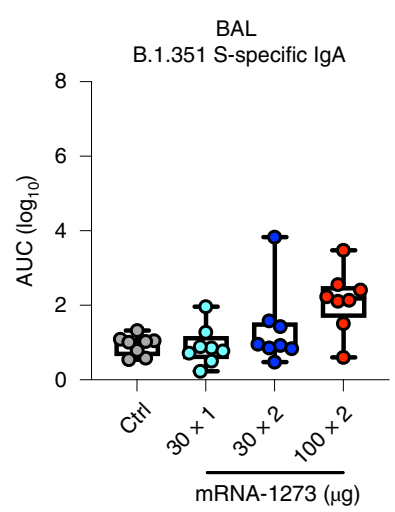

j
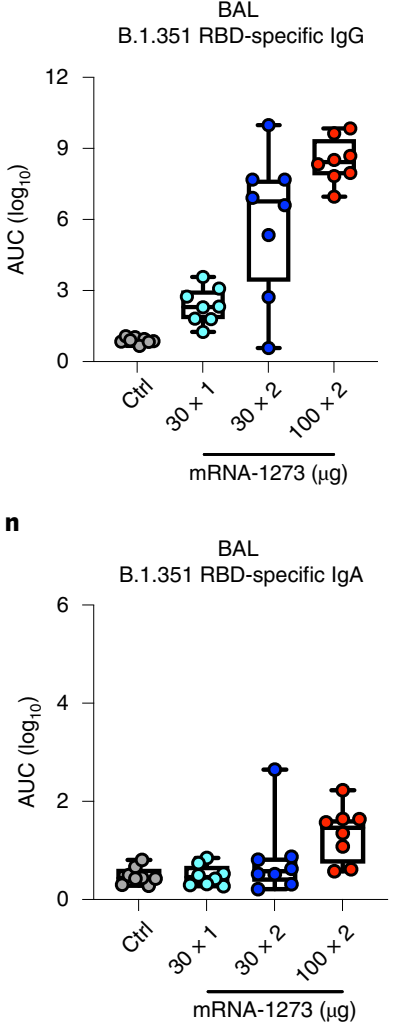

c
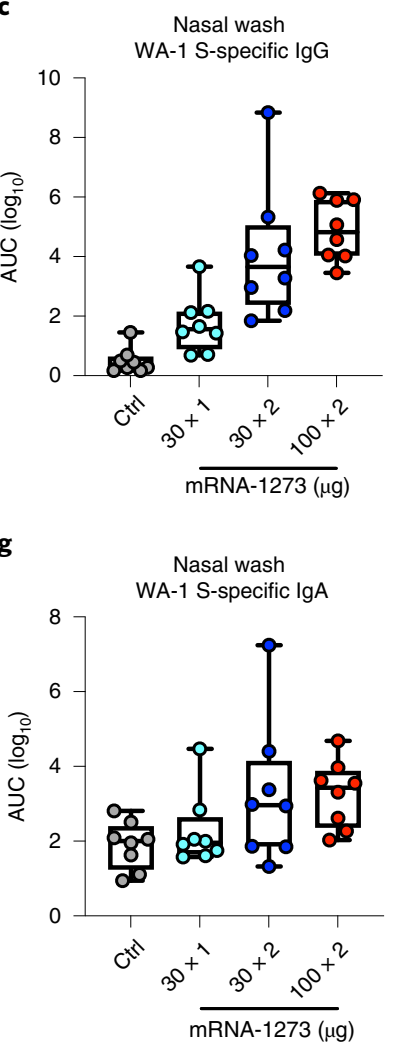

k

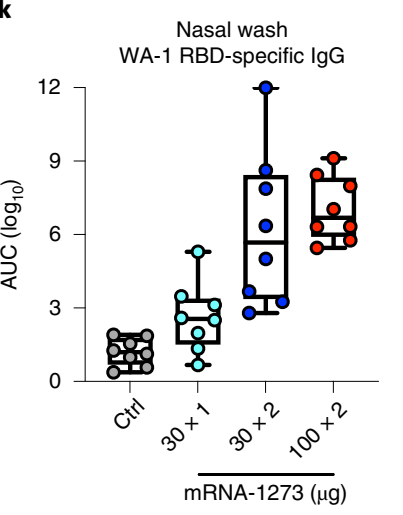

o

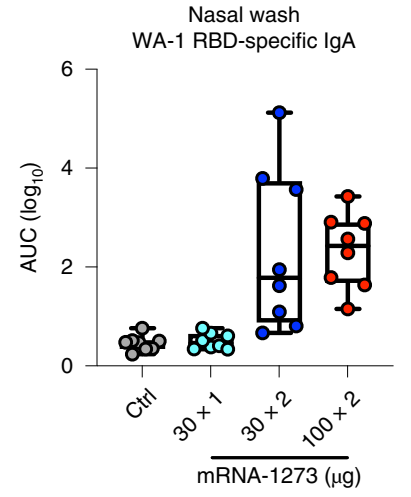

d

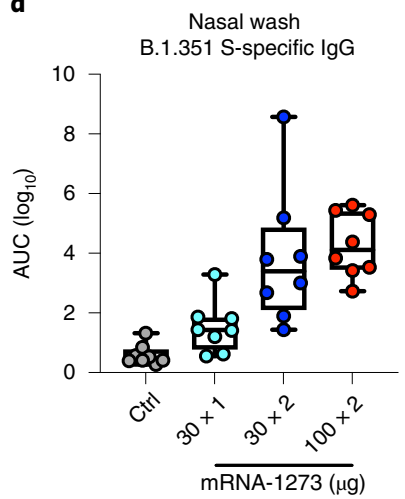

h

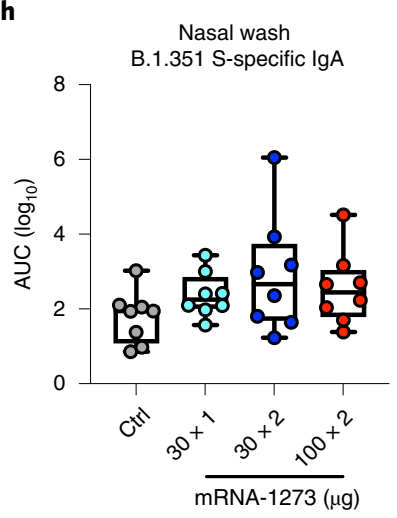

I

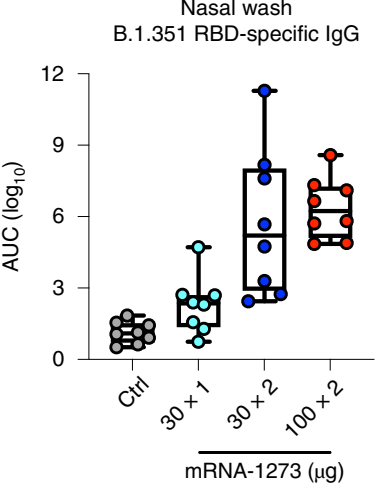

p

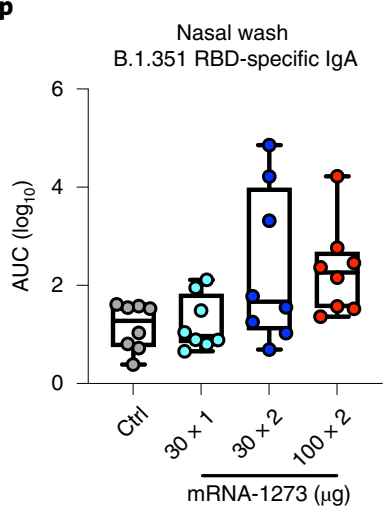

Fig. 2 | Mucosal antibody responses following mRNA-1273 immunization. a-p, Twenty-four rhesus macaques were immunized according to Extended Data Fig. 1, and compared to eight age-matched controls. BAL (a, $\mathbf{b}, \mathbf{e}, \mathbf{f}, \mathbf{i}, \mathbf{j}, \mathbf{m}$ and $\mathbf{n}$ ) and nasal washes (c, $\mathbf{d}, \mathbf{g}, \mathbf{h}, \mathbf{k}, \mathbf{I}, \mathbf{o}$ and $\mathbf{p})$ collected at week 7 were assessed for SARS-CoV-2 WA-1 (a, c, e, g, i, $\mathbf{k}, \mathbf{m}$ and $\mathbf{o}$ ) and B.1.351 (b, d, f, h, j, I, $\mathbf{n}$ and $\mathbf{p}$ ) S-specific (a-h) and RBD-specific (i-p) IgG (a-d and i-I) and IgA (e-h and $\mathbf{m}-\mathbf{p})$ by MULTI-ARRAY ELISA. Data represent one independent experiment. Circles represent individual NHPs. Boxes and horizontal bars denote the IQR and medians, respectively; whisker end points are equal to the maximum and minimum values. 
a

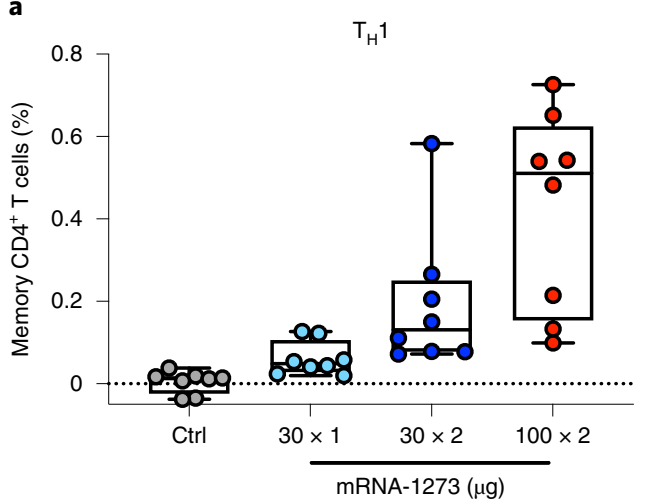

c
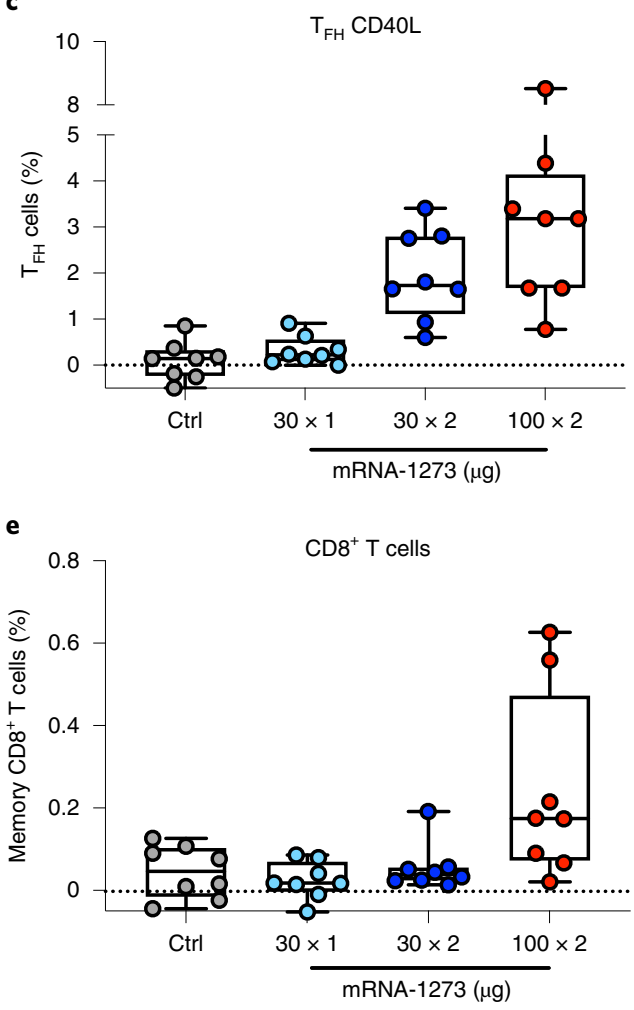

b

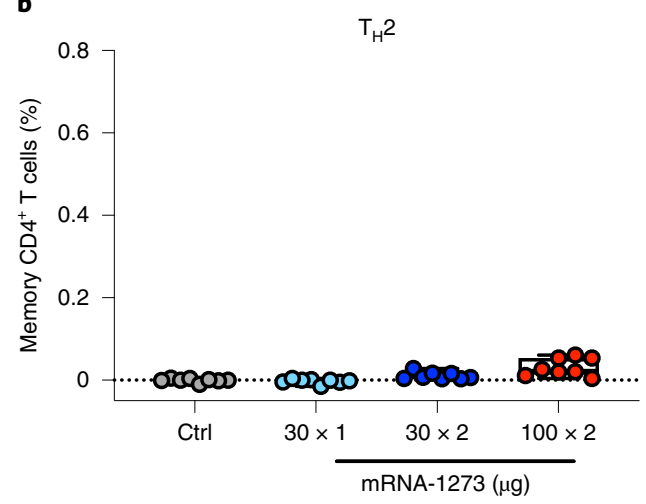

d

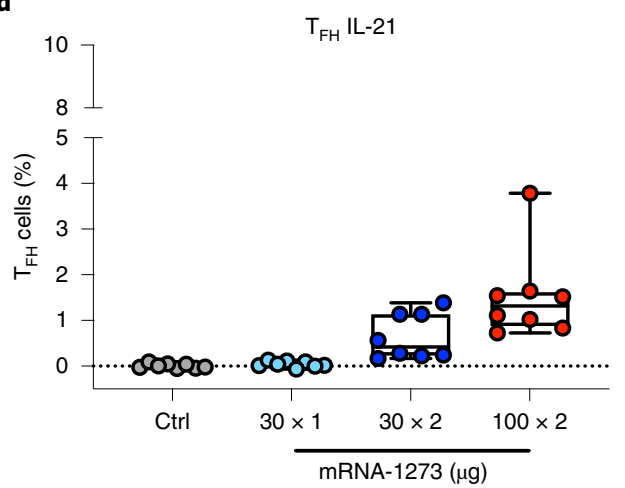

Fig. 3 | T cell responses following mRNA-1273 immunization. a-e, Twenty-four rhesus macaques were immunized according to Extended Data Fig. 1, and compared to eight age-matched controls. Intracellular staining was performed on peripheral blood mononucleolar cells at week 7 to assess $T$ cell responses to SARS-CoV-2 S-protein peptide pools, S1 and S2. Responses to S1 and S2 individual peptide pools were summed. a, $\mathrm{T}_{H} 1$ responses (interferon (IFN)- $\gamma$, IL-2 or tumor necrosis factor). b, $T_{H} 2$ responses (IL-4 or IL-13). $\mathbf{c}_{,} \mathrm{T}_{\mathrm{FH}}$ CD40L upregulation (peripheral $\mathrm{T}_{\mathrm{FH}}$ cells were gated on central memory CXCR5 + PD-1+ICOS+ CD4+ T cells). d, $\mathrm{T}_{\mathrm{FH}}$ IL-21. e, CD8 ${ }^{+} \mathrm{T}$ cells. Data represent one independent experiment. Boxes and horizontal bars denote the IQR and medians, respectively; whisker end points are equal to the maximum and minimum values. Circles represent individual NHPs. Dotted lines are set to $0 \%$.

performed after each passage $(\mathrm{P})$ of the B.1.351 strain, and the challenge stock retained all mutations in the RBD and NTD sites on the spike protein (Extended Data Fig. 4a). The John Hopkins University (JHU) B.1.351 P2 stock was then administered to golden Syrian hamsters, a highly pathogenic SARS-CoV-2 animal model, at three different concentrations to characterize weight loss (Extended Data Fig. 4b) and in NHPs to measure upper and lower airway viral replication by quantitative PCR with reverse transcription (RT-qPCR) for subgenomic RNA (sgRNA; Extended Data Fig. 4c,d). Based on these data, a B.1.351 challenge dose of $5 \times 10^{5}$ plaque-forming units (PFUs) was selected for the vaccine study; this dose would induce sgRNA levels similar to the higher values obtained from nasal secretions of humans following SARS-CoV-2 infection ${ }^{43,44}$.
To assess the protective efficacy of mRNA-1273 against the B.1.351 SARS-CoV-2 variant, the NHPs were challenged with a total dose of $5 \times 10^{5}$ PFUs of B.1.351 by intratracheal and intranasal routes 7-8 weeks after boost for the two-dose regimens and 12 weeks after the single-dose regimen. Two days after challenge, only two of eight NHPs that received $100 \mu \mathrm{g} \times 2$ of mRNA-1273 had detectable SARS-CoV-2 envelope (E) sgRNA (sgRNA_E) in BAL compared to all NHPs in the control group (Fig. 4a). By RT-qPCR of sgRNA_E in BAL (Fig. 4a) or nucleocapsid (N) sgRNA (sgRNA_N) in BAL (Fig. 4c), the $100-\mu \mathrm{g} \times 2$ group had a significant decrease in viral load compared to NHPs that received a single dose of $30 \mu \mathrm{g}(P=0.0054)$ or to the control NHPs $(P=0.0009)$. NHPs that received $30 \mu \mathrm{g} \times 2$ also had a significant decrease in viral load compared to control NHPs $(P=0.0054)$ and showed a trend toward 
reduced viral replication compared to a single immunization with $30 \mu \mathrm{g}$ (Fig. $4 \mathrm{a}, \mathrm{c}$ ). NHPs that received a single vaccination with $30 \mu \mathrm{g}$ showed a trend toward reduced viral replication compared to control NHPs (Fig. 4a,c). On day 4 after challenge, the pattern was the same, with a significant reduction in viral replication in BAL for all vaccine groups compared to control NHPs, and for the $100-\mu \mathrm{g}$ group compared to the $30-\mu \mathrm{g} \times 1$ group (Fig. $4 \mathrm{a}, \mathrm{c}$ ). By day 7 , while seven of eight control NHPs still had $\sim 4$ logs of sgRNA_E, there was no detectable sgRNA_E in six of eight NHPs in all vaccine groups (Fig. 4a), consistent with control of viral replication in the lower airway. To extend the analysis, we assessed viral titers in BAL and nasal swab (NS) samples following challenge (Fig. 4e,h). There was low to undetectable virus cultured from BAL in the majority of NHPs immunized with 30 and $100 \mu \mathrm{g} \times 2$ at day 2 after challenge (Fig. $4 \mathrm{e}$ ). Moreover, on day 2 after challenge, BAL viral titers and sgRNA were highly correlated (Fig. 4f,g), where there was no culturable virus from BAL of all NHPs with BAL sgRNA_N $<1.2 \times 10^{4}$ RNA copies per ml (Fig. 4g).

In contrast to the significant reduction of viral replication in the lower airway, at day 2 after challenge, the only significant reduction in viral replication for sgRNA_E (Fig. 4b) or sgRNA_N (Fig. 4d) in NS samples was in the 100- $\mu$ g group. The differences in NHPs that received $100 \mu \mathrm{g} \times 2$ were marginally lower compared to the groups that received $30 \mu \mathrm{g} \times 2$ or $30 \mu \mathrm{g} \times 1 \quad(P=0.0273$ and 0.0350 , respectively), with no other significant pairwise differences (Fig. 4b,d). The groups were not significantly different at the later time points. A notable finding was that five of eight control NHPs still had $\sim 4$ $\log _{10}$ of sgRNA_E and all eight NHPs had sgRNA_N at day 7 in the NS samples (Fig. 4b,d), highlighting persistence of sgRNA following B.1.351 through day 7 after challenge. With that, there was $\sim 4 \log _{10}$ of sgRNA_E at day 7 in the NS samples of three of eight NHPs in the $100-\mu \mathrm{g} \times 2$ group (Fig. $4 \mathrm{~b}$ ). Of note, six of eight animals had low to undetectable viral load in NS samples on day 2 after challenge in the $100-\mu \mathrm{g} \times 2$ group (Fig. $4 \mathrm{~h}$ ). NS viral titers and sgRNA were highly correlated (Fig. 4i,j). Overall, these data show a significant reduction and rapid control of B.1.351 viral replication in the lower airways following vaccination with mRNA-1273 with more limited control in the upper airway and only in the $100-\mu \mathrm{g} \times 2$ group.

Inflammation and viral load in lung tissue after challenge. To provide a further assessment of protection following vaccination, NHPs in each of the dose groups were assessed for virus-related pathology and the detection of viral antigen in the lung $8 \mathrm{~d}$ after B.1.351 challenge. The severity of inflammation, which ranged from minimal to moderate, was similar across lung samples from NHPs that received the vaccine in doses of $100 \mu \mathrm{g} \times 2,30 \mu \mathrm{g} \times 2$ or $30 \mu \mathrm{g} \times 1$ (Fig. 5). The inflammatory lesions in the lung were characterized by a mixture of lymphocytes, histiocytes and fewer polymorphonuclear cells associated with variably expanded alveolar capillaries, occasional areas of perivascular inflammation and type II pneumocyte hyperplasia. Two of four NHPs that received $30 \mu \mathrm{g} \times 1$ of mRNA-1273 vaccine had trace amounts of virus detected in the lung. There was no detection of viral antigen in any lung sample from NHPs that received two doses of 30 or $100 \mu \mathrm{g}$. All four NHPs in the control group had variable amounts of viral antigen detected in the lung (Fig. 5 and
Supplementary Table 1). These data corroborate viral replication data suggesting two doses of mRNA-1273 elicits substantial protection against lower-airway disease following B.1.351 challenge.

Post-challenge antibody and $\mathrm{T}$ cell responses in the airways. The assessment of antibody responses after challenge has been useful for determining whether viral replication in the BAL or NS samples is sufficient to boost vaccine-induced anamnestic S-specific antibody responses in these mucosal tissues ${ }^{38,45}$. In BAL samples, WA- 1 and B.1.351 S-specific IgG (Extended Data Fig. 5a,b) or IgA (Extended Data Fig. 5c,d) responses did not increase after challenge in NHPs that received two immunizations of 30 or $100 \mu \mathrm{g}$ of mRNA-1273. However, by $14 \mathrm{~d}$ after challenge, there was an increase in WA- 1 and B.1.351 S-specific IgG responses in NHPs that received $30 \mu \mathrm{g} \times 1$ of mRNA-1273; in these NHPs IgG titers increased to levels that were similar to the NHPs that received 30 or $100 \mu \mathrm{g} \times 2$ and higher than the unvaccinated controls (Extended Data Fig. 5a,b). In NS samples, there was an increase in WA-1 and B.1.351 S-specific IgG (Extended Data Fig. 5e,f) or IgA (Extended Data Fig. 5g,h) responses in unvaccinated NHPs and those immunized with $30 \mu \mathrm{g}$ of mRNA-1273 once or twice; however, there were no anamnestic S-specific antibody responses in the $100-\mu$ group. Overall, these data show that the increase in S-specific anamnestic antibody responses in both the BAL and NS samples are associated with viral replication in these mucosal sites and may explain the relatively rapid clearance of virus from NS samples in the $30-\mu \mathrm{g} \times 1$ group seen from days 4 to 7 (Fig. 4b,d). We also assessed anamnestic S-specific T cell responses in BAL samples following challenge. $\mathrm{CD}^{+} \mathrm{T}$ cell responses, and not $\mathrm{CD}^{+} \mathrm{T}$ cells, were increased after challenge in BAL samples in $30-\mu \mathrm{g}$ $\times 1$ mRNA-1273-immunized NHPs and control NHPs (Extended Data Fig. 6). These data are consistent with their increased viral load in BAL samples compared to the two-dose vaccine groups.

Antibody correlates of protection. Assessing immune correlates of protection following vaccination is a critical aspect of vaccine development. It was reported that mRNA-1273 induced antibody responses are a mechanistic correlate for reducing viral replication against WA-1 challenge in NHPs ${ }^{38}$. Here, B.1.351 S-specific IgG antibody titers at week 12, the time of challenge, also correlated strongly with reduction of sgRNA in both BAL (Fig. 6a) and NS (Fig. 6d) samples at day 2 after challenge. In addition, both pseudovirus and live viral neutralization correlated significantly with reduction of sgRNA in both BAL (Fig. 6b,c) and NS (Fig. 6e,f) samples.

In a recent report on correlates of protection following mRNA1273 immunization in NHPs, we established a linear relationship between WA-1 S-specific antibody titers as defined by international units (IUs) and subsequent sgRNA $2 \mathrm{~d}$ after SARS-CoV-2 WA- 1 challenge. Here, we similarly converted pre-challenge WA-1 S-specific antibody titers to IUs (Supplementary Table 2). Consistent with our previous NHP study, pre-challenge WA-1 S-specific IgG titers and sgRNA in BAL (Fig. 6g) and NS (Fig. 6h) samples at day 2 after challenge were negatively correlated. Based on a linear model, vaccinated animals that had S-specific IgG titers of $\sim 100$ and $120 \mathrm{IU} \mathrm{ml}^{-1}$ were predicted to have sgRNA_N and sgRNA_E, respectively, in BAL samples of approximately $2 \log _{10}$ lower than the average for

Fig. 4 | Efficacy of mRNA-1273 against upper and lower respiratory B.1.351 viral replication. a-j, Twenty-four rhesus macaques were immunized and challenged as described in Extended Data Fig. 1, and compared to eight age-matched controls. BAL ( $\mathbf{a}$ and $\mathbf{c}$ ) and NS (b and $\mathbf{d}$ ) samples were collected on days 2 (circles), 4 (squares), 7 (triangles) and 14 (inverted triangles) after challenge, where applicable, and viral replication was assessed by detection of SARS-CoV-2 E-specific ( $\mathbf{a}$ and $\mathbf{b}$ ) and N-specific ( $\mathbf{c}$ and $\mathbf{d}$ ) sgRNA. Viral titers were assessed by a $50 \%$ tissue culture infectious dose $\left(\mathrm{TCID}_{50}\right.$ ) assay for BAL collected on days 2 and 4 after challenge (e) and for NS on day 2 (h). Boxes and horizontal bars denote the IQR and medians, respectively; whisker end points are equal to the maximum and minimum values. In $\mathbf{f}, \mathbf{g}$, $\mathbf{i}$, and $\mathbf{j}$, plots show correlations between viral titers and sgRNA_E (f and $\mathbf{i}$ ) and sgRNA_N ( $\mathbf{g}$ and $\mathbf{j}$ ) in BAL ( $\mathbf{f}$ and $\mathbf{g}$ ) and NS (i and $\mathbf{j}) 2 \mathrm{~d}$ after challenge. Data represent one independent experiment. Black and gray lines indicate linear regression line and 95\% confidence interval, respectively. $r$ and $P$ represent Spearman's correlation coefficients and corresponding two-sided $P$ values, respectively; all $P$ values were $<0.0001$. Symbols represent individual NHPs and may overlap for equal values. 
PBS controls. Serum neutralizing activity was associated with control of viral replication; 13 of 14 of the NHPs with detectable pseudovirus neutralizing activity against B.1.351 had BAL sgRNA_N $<10^{5}$
(Fig. 6b). A similar linear relationship between S-specific antibody titers and viral replication in NS samples was apparent (Fig. 6e), although few of the NHP had NS sgRNA_N below $10^{5}$. a

BAL sgRNA_E

Days after B.1.351 challenge

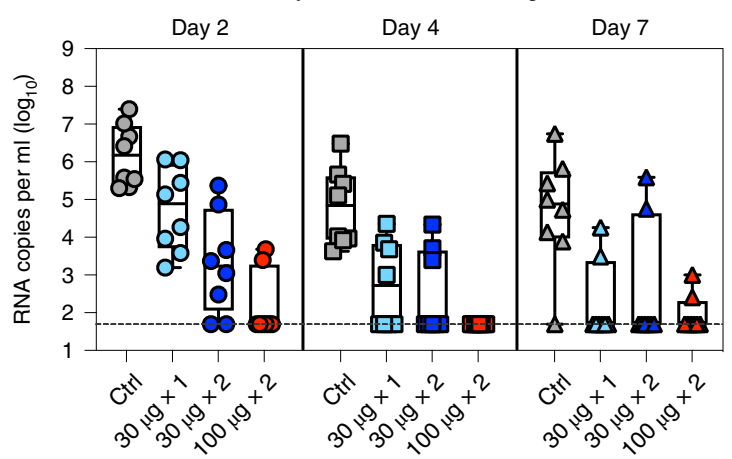

C

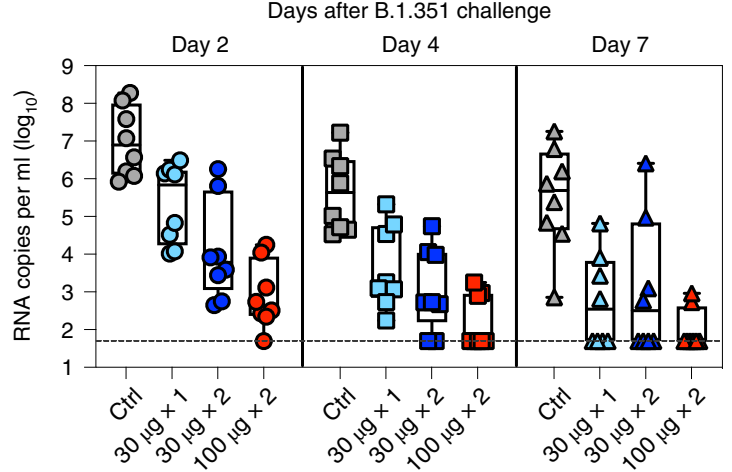

Viral titers in BAL

Days after B.1.351 challenge

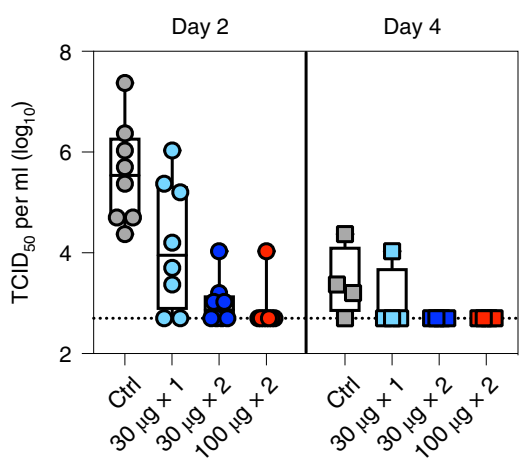

h

Viral titers in NS

Days after B.1.351 challenge

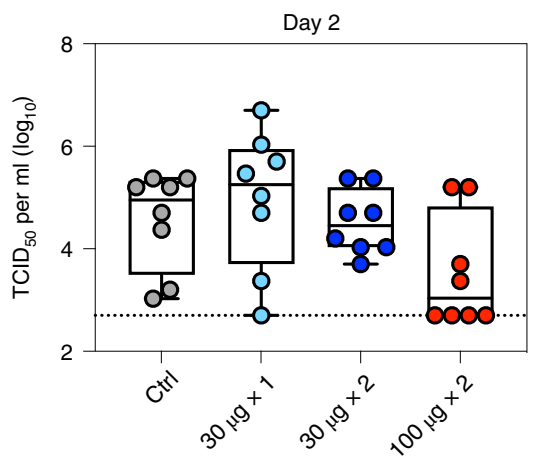

i b Days after B.1.351 challenge

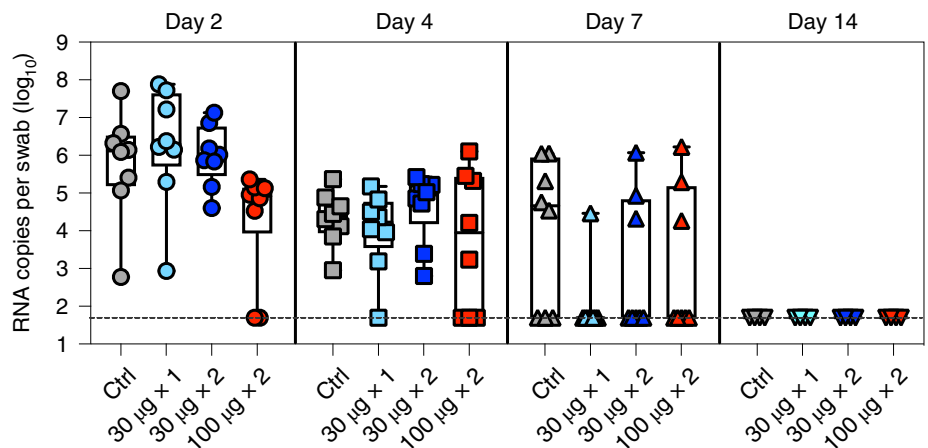

NS sgRNA_N

Days after B.1.351 challenge

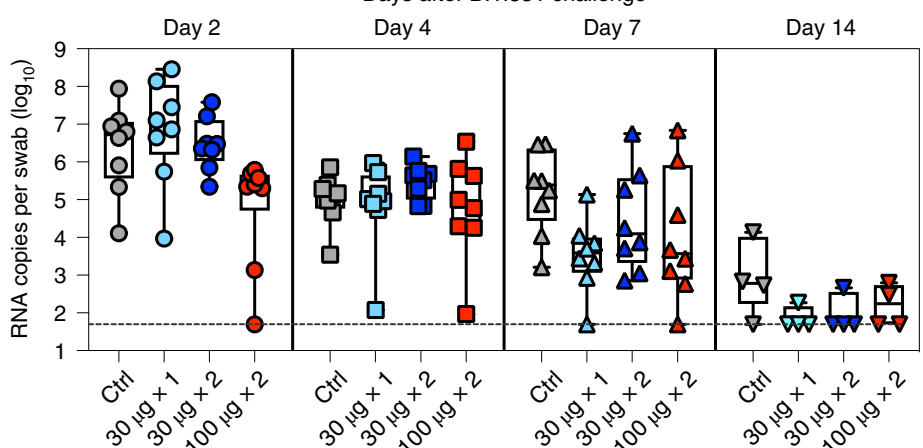

g

BAL viral titer vs. BAL sgRNA_E

Day 2
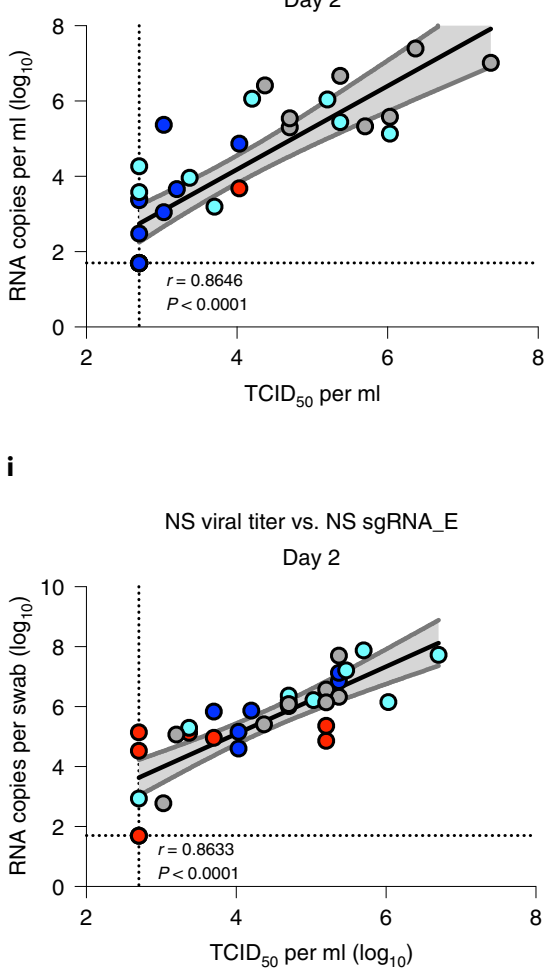
Day 2

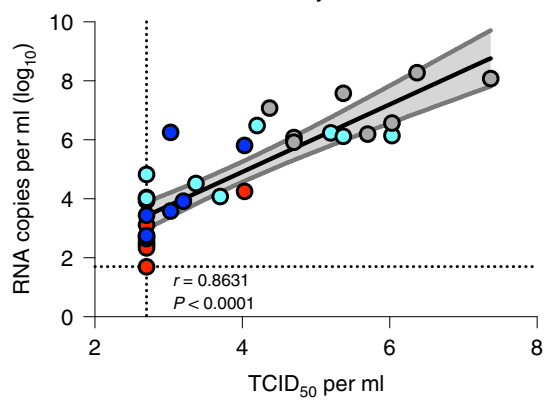

j

NS viral titer vs. NS sgRNA_N Day 2

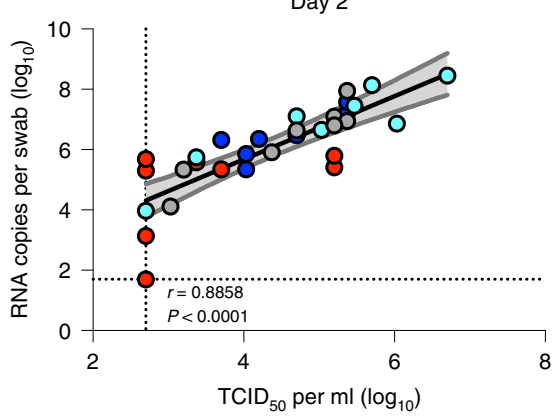


As the antibody titers of S-specific IgG are very similar to those we previously reported using NHPs challenged with the SARS-CoV-2 WA-1 (ref. ${ }^{38}$ ), we conducted an additional exploratory analysis of the data from both studies to investigate the consistency of the relationship between S-specific antibodies and BAL sgRNA, across the two different viruses. For low S-specific IgG levels, sgRNA was higher for animals challenged with B.1.351, but for animals with titers of S-specific IgG greater than approximately $100 \mathrm{IU} \mathrm{ml}^{-1}$, the estimated regression curve slopes are similar as are the levels that correspond to 2 - to $4-\log _{10}$ reductions in sgRNA in BAL samples compared to controls (Fig. 6i). These data suggest a similar relationship between S-specific IgG titers and lower-airway protection against WA-1, which contains $S$ protein that is homologous to the mRNA-1273 insert, and the B.1.351 variant SARS-CoV-2 strain in NHPs.

\section{Discussion}

mRNA-1273 and BNT162b2 vaccines had $~ 95 \%$ efficacy in clinical trials performed in the United States, when WA-1 and p.Asp614Gly variants circulated most widely ${ }^{46,47}$. A critical issue is whether these and other vaccines will mediate protection against rapidly emerging variants. The B.1.351 variant is one of greatest concern compared to WA-1, p.Asp614Gly or B.1.1.7 based on the higher reduction in neutralization using vaccine sera ${ }^{4-11,24,25,48}$ and clinical trials showing lower efficacy against symptomatic infection ${ }^{29,30,32}$. However, recent data show the B.1.617.2 variant is now the predominant circulating strain worldwide, although it is less resistant to neutralization than B.1.351. Here, we present evidence that vaccine dose and number of immunizations effect protection against B.1.351 challenge. These data are consistent with a recent report showing vaccine effectiveness against PCR-confirmed infection with the B.1.351 variant is $16 \%$ after one dose of BNT162b2 and 75\% after two doses ${ }^{31}$.

Antibodies have a critical role in mediating vaccine-elicited protection against SARS-CoV-2 in NHP models ${ }^{33,37,39,45,49}$. Here, we show there was a dose-dependent increase in WA-1 and B.1.351 S-specific and RBD-specific antibody titers and p.Asp614Gly and B.1.351 neutralization titers. Neutralizing activity against B.1.351 and B.1.617.2 were both increased following the second immunization. These data highlight the importance of a two-dose regimen for optimizing neutralization antibody responses, particularly against B.1.351 and likely for other VOCs with increased neutralization resistance. The frequency of S-specific $\mathrm{T}_{\mathrm{H}} 1$ and $\mathrm{T}_{\mathrm{FH}}$ cell responses were also dose dependent, and $\mathrm{CD} 8^{+} \mathrm{T}$ cell responses detected only in NHPs receiving the $100-\mu \mathrm{g}$ dose. These data are consistent with previous studies by us and others showing mRNA is a vaccine platform that induces $\mathrm{T}_{\mathrm{FH}}$ cells and robust binding and neutralizing antibodies $^{33,38,41,50}$.

As this study was our first to use the B.1.351 variant for challenge in NHPs, extensive sequence analyses were performed to propagate a challenge stock with an $\mathrm{S}$ sequence matched to the reference isolate. Naive NHPs infected with the B.1.351 stock notably had peak sgRNA levels of $\sim 10^{7}$ copies per $\mathrm{ml}$ in BAL samples, which is higher than reported by us and others for challenge studies using the WA-1 strain $^{33,37,39,45,49}$. Seven days after challenge, most of the control NHPs still had $\sim 10^{5}$ RNA copies per $\mathrm{ml}$ and copies per swab present in BAL and NS samples, respectively. This contrasts with the more rapid and complete reduction of viral replication following challenge with the WA-1 $\operatorname{strain}^{33,37,39,45,49}$ and is higher than viral loads typically seen in human infections. Whether the amount and persistence of the B.1.351 virus in vivo in this study relates to the challenge dose or suggests that this variant has inherent properties that make it more difficult to control and clear compared to the WA- $1 \operatorname{strain}^{51,52}$ is a focus of ongoing analyses.

Based on the high levels of sgRNA in BAL and NS samples, the dose of B.1.351 used here provided a stringent challenge for vaccine-elicited protection. We observed a significant reduction in
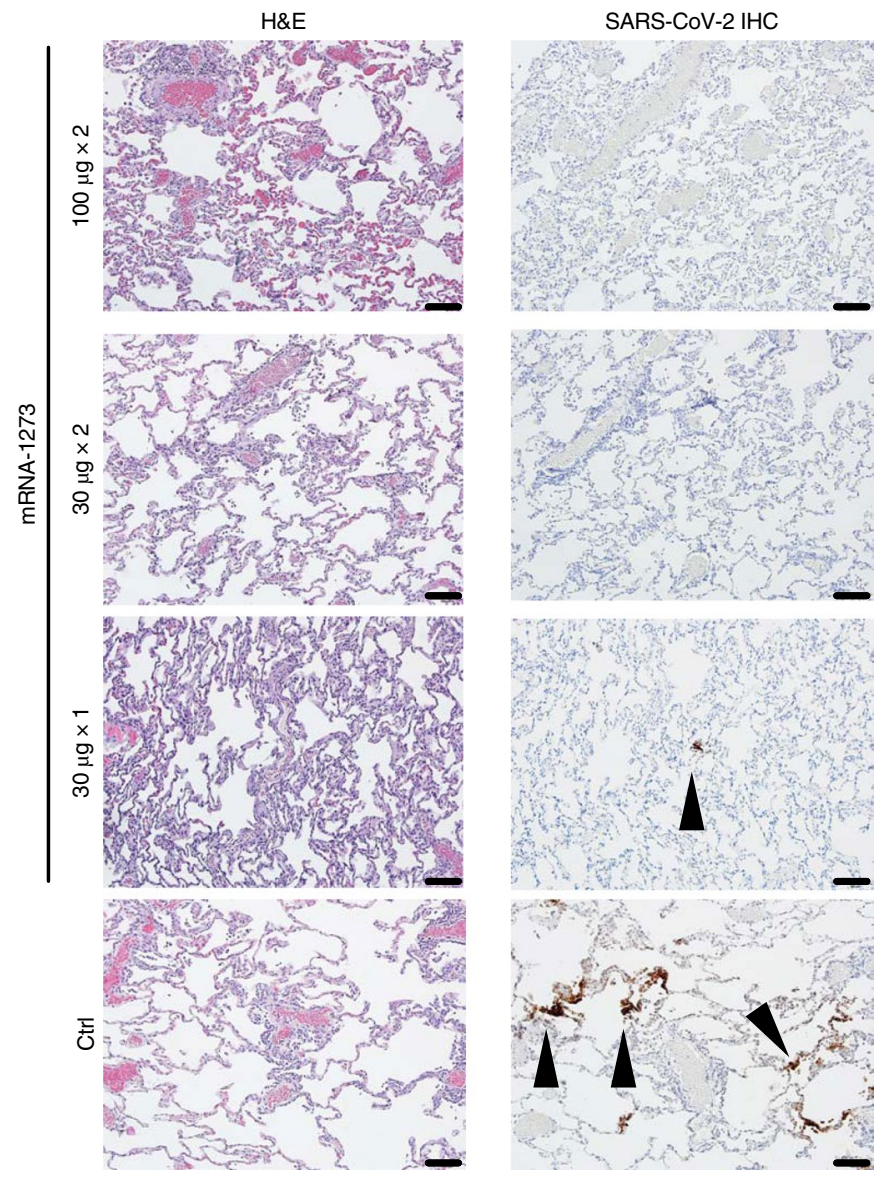

Fig. 5 | Post-challenge lung histopathological analysis and viral detection. Rhesus macaques were immunized and challenged as described in Supplementary Fig. 1. Eight days after challenge, lung samples ( $n=4$ per group) were evaluated for the presence of inflammation by hematoxylin and eosin (H\&E) staining (left) and evidence of virus infection by immunohistochemistry (IHC) for SARS-CoV-2 viral antigen (right). Representative images show the location and distribution of SARS-CoV-2 viral antigen in serial lung tissue sections. Arrowheads indicate areas positive for viral antigen. Each image is taken at $\times 10$ magnification; scale bars, $100 \mu \mathrm{m}$.

viral replication in BAL samples at day 2 after challenge in groups that received 30 and $100 \mu \mathrm{g}$ twice; six of eight NHPs that received the 100- $\mu$ g vaccine regimen had no detectable sgRNA_E in BAL samples. Of note, while there was no detectable serum neutralizing activity against B.1.351 virus with a single immunization of $30 \mu \mathrm{g}$, there was still a significant reduction in viral replication in BAL samples by day 4 after challenge compared to controls, with limited inflammation or viral antigen in lungs at day 8 . These data are consistent with our recent study in which NHPs immunized with only 1 or $3 \mu \mathrm{g}$ of mRNA-1273 twice demonstrated a reduction in viral replication in BAL samples and limited lung pathology following WA-1 challenge despite absence of detectable neutralizing activity ${ }^{38}$. Potential mechanisms for lower-airway protection in the absence of detectable serum neutralizing antibodies include Fc effector functions and anamnestic responses after challenge. It is also possible that $\mathrm{T}$ cells contribute to control of viral replication in lower airways; however, no current evidence exists for viral control by $\mathrm{T}$ cells following vaccination or primary infection ${ }^{53}$ in the SARS-CoV-2 NHP model.

In contrast to high-level or complete protection observed in lower airways against B.1.351 challenge for all doses, control of 

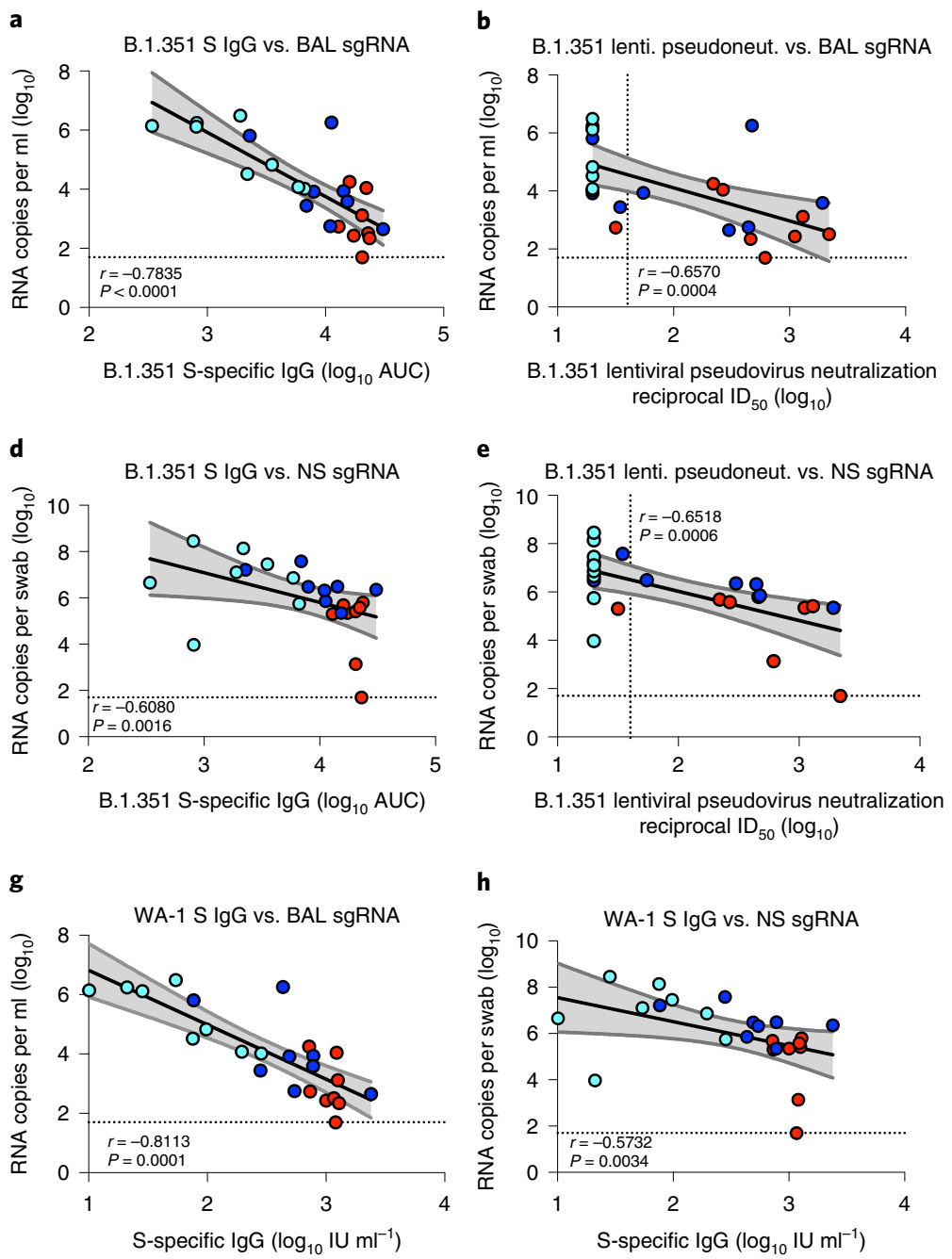
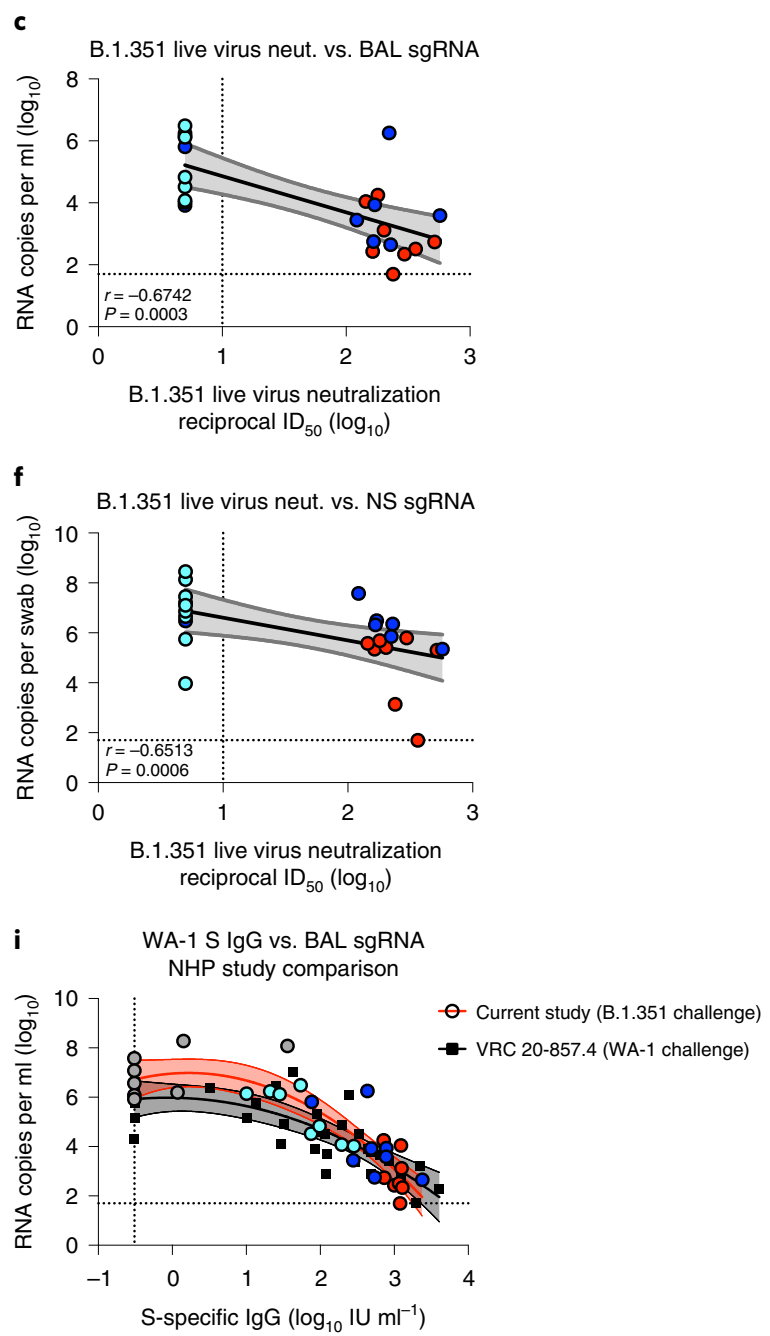

Fig. 6 | Antibody correlates of protection. a-i, Twenty-four rhesus macaques were immunized and challenged as described in Extended Data Fig. 1, and compared to eight age-matched controls. a-f, Plots show correlations and the corresponding two-sided $P$ values between week-12 SARS-CoV-2 B.1.351 S-specific IgG (a and $\mathbf{d}$ ), lentiviral-based pseudovirus neutralization (b and $\mathbf{e}$ ), and focus-reduction live-virus neutralization (c and $\mathbf{f}$ ) with $\mathrm{N}$-specific sgRNA in BAL (a-c) and NS (d-f) samples at day 2 after challenge. $\mathbf{g}, \mathbf{h}$, Plots show correlations and the corresponding two-sided $P$ values between week-12 SARS-CoV-2 WA-1 S-specific IgG, converted to IU $\mathrm{ml}^{-1}$, with N-specific sgRNA in BAL (g) and NS (h) at day 2 after challenge. Circles represent individual NHPs, where colors indicate mRNA-1273 dose. Dotted lines indicate assay limits of detection. i, The relationship between pre-challenge WA-1 S-specific IgG and day-2 BAL sgRNA_N, with data from the current study using a B.1.351 challenge (filled circles, red curve fit) superimposed on data from our previous study with a WA-1 challenge (black squares, black curve fit); lines indicate quadratic curve fit and $95 \%$ confidence intervals. Data represent one independent experiment. Symbols represent individual NHPs and may overlap for equal values. VRC, Vaccine Research Center.

viral replication in upper airways was only observed in animals receiving two doses of $100 \mu \mathrm{g}$. These data are consistent with previous studies in NHPs showing higher amounts of antibody are required to reduce viral replication in upper than lower airways following mRNA-1273 vaccination ${ }^{33,38}$. The implications of these findings on transmission of B.1.351 in vaccinated people are unknown. Of note, recent results from human vaccine trials show that there is greater protection against severe disease than mild disease against the B.1.351 variant following immunization with Ad26.CoV2 (ref. ${ }^{29}$ ) or BNT162b2 (ref. ${ }^{31}$ ). Serum antibody titers were strong predictors of reduced viral load in BAL and NS samples. These data are consistent with studies by us and others showing antibodies induced by vaccination ${ }^{38}$ or infection ${ }^{53}$ of NHPs protect against reinfection. Our modeling suggests that animals with S-specific IgG titers of approximately $100 \mathrm{IU} \mathrm{m}^{-1}$ reduce sgRNA levels in BAL samples by approximately $2 \log _{10}$, with further decrease of approximately $2 \log _{10}$ sgRNA for every $1-\log _{10}$ increase in IgG titer in IU ml- $\mathrm{me}^{-1}$. We also noted that 13 of 14 animals with any detectable neutralizing antibodies against B.1.351 had BAL sgRNA_N $<10^{5}$ suggesting that vaccines eliciting low to undetectable B.1.351-specific neutralizing antibodies may still control viral replication in lower airways.

The NHP model has been critical for guiding vaccine development against COVID-19 in humans. This report provides evidence that a two-dose regimen with mRNA-1273 effectively protects against B.1.351. Importantly, these data show a two-dose regimen induces high neutralizing activity against the B.1.617.2 VOC and ongoing studies will determine if it is sufficient to protect NHPs. A final issue is durability of immunity and protection following vaccination. Ongoing studies assess how additional boosting with WA-1 or variant strain spike antigens influence protection against B.1.351 and other variants in NHPs and humans. These data in conjunction with real-world clinical data will inform public health decisions on future vaccine boost requirements. 


\section{Online content}

Any methods, additional references, Nature Research reporting summaries, source data, extended data, supplementary information, acknowledgements, peer review information; details of author contributions and competing interests; and statements of data and code availability are available at https://doi.org/10.1038/ s41590-021-01021-0.

Received: 23 June 2021; Accepted: 4 August 2021; Published online: 20 August 2021

\section{References}

1. Supasa, P. et al. Reduced neutralization of SARS-CoV-2 B.1.1.7 variant by convalescent and vaccine sera. Cell 184, 2201-2211 (2021).

2. Chen, R. E. et al. Resistance of SARS-CoV-2 variants to neutralization by monoclonal and serum-derived polyclonal antibodies. Nat. Med. 27, 717-726 (2021).

3. Edara, V. V. et al. Infection- and vaccine-induced antibody binding and neutralization of the B.1.351 SARS-CoV-2 variant. Cell Host Microbe 29, 516-521 (2021).

4. Li, Q. et al. The impact of mutations in SARS-CoV-2 spike on viral infectivity and antigenicity. Cell 182, 1284-1294 (2020).

5. Tegally, H. et al. Detection of a SARS-CoV-2 variant of concern in South Africa. Nature https://doi.org/10.1038/s41586-021-03402-9 (2021).

6. Wibmer, C. K. et al. SARS-CoV-2 501Y.V2 escapes neutralization by South African COVID-19 donor plasma. Nat. Med. 27, 622-625 (2021).

7. Voysey, M. et al. Safety and efficacy of the ChAdOx1 nCoV-19 vaccine (AZD1222) against SARS-CoV-2: an interim analysis of four randomised controlled trials in Brazil, South Africa and the UK. Lancet 397, 99-111 (2021).

8. Voysey, M. et al. Single-dose administration and the influence of the timing of the booster dose on immunogenicity and efficacy of ChAdOx1 nCoV-19 (AZD1222) vaccine: a pooled analysis of four randomised trials. Lancet 397, 881-891 (2021).

9. Andreano, E. et al. SARS-CoV-2 escape in vitro from a highly neutralizing COVID-19 convalescent plasma. Preprint at https://doi.org/10.1101/2020. 12.28.424451 (2020).

10. Hoffmann, M. et al. SARS-CoV-2 variants B.1.351 and P.1 escape from neutralizing antibodies. Cell 184, 2384-2393 (2021).

11. Garcia-Beltran, W. F. et al. Multiple SARS-CoV-2 variants escape neutralization by vaccine-induced humoral immunity. Cell https://doi. org/10.1016/j.cell.2021.03.013 (2021).

12. Tada, T. et al. Decreased neutralization of SARS-CoV-2 global variants by therapeutic anti-spike protein monoclonal antibodies. Preprint at https://doi org/10.1101/2021.02.18.431897 (2021).

13. Tada, T. et al. Neutralization of viruses with European, South African and United States SARS-CoV-2 variant spike proteins by convalescent sera and BNT162b2 mRNA vaccine-elicited antibodies. Preprint at https://doi. org/10.1101/2021.02.05.430003 (2021).

14. Deng, X. et al. Transmission, infectivity, and neutralization of a spike L452R SARS-CoV-2 variant. Cell. https://doi.org/10.1016/j.cell.2021.04.025 (2021).

15. Greaney, A. J. et al. Comprehensive mapping of mutations in the SARS-CoV-2 receptor-binding domain that affect recognition by polyclonal human plasma antibodies. Cell Host Microbe 29, 463-476 (2021).

16. McCallum, M. et al. N-terminal domain antigenic mapping reveals a site of vulnerability for SARS-CoV-2. Cell https://doi.org/10.1016/j.cell.2021.03.028 (2021).

17. Wang, P. et al. Antibody resistance of SARS-CoV-2 variants B.1.351 and B.1.1.7. Nature https://doi.org/10.1038/s41586-021-03398-2 (2021).

18. Zhou, H. et al. B.1.526 SARS-CoV-2 variants identified in New York City are neutralized by vaccine-elicited and therapeutic monoclonal antibodies. mBio. https://doi.org/10.1128/mBio.01386-21 (2021).

19. Liu, C. et al. Reduced neutralization of SARS-CoV-2 B.1.617 by vaccine and convalescent serum. Cell https://doi.org/10.1016/j.cell.2021.06.020 (2021).

20. Pegu, A. et al. Durability of mRNA-1273 vaccine-induced antibodies against SARS-CoV-2 variants. Science. https://doi.org/10.1126/science.abj4176 (2021).

21. Tegally, H. et al. Detection of a SARS-CoV-2 variant of concern in South Africa. Nature. https://doi.org/10.1038/s41586-021-03402-9 (2021).

22. Li, Q. et al. SARS-CoV-2 501Y.V2 variants lack higher infectivity but do have immune escape. Cell 184, 2362-2371 (2021).

23. Zhou, D. et al. Evidence of escape of SARS-CoV-2 variant B.1.351 from natural and vaccine-induced sera. Cell 184, 2348-2361 (2021)

24. Starr, T. N. et al. Deep mutational scanning of SARS-CoV-2 receptor-binding domain reveals constraints on folding and ACE2 binding. Cell $\mathbf{1 8 2}$, 1295-1310 (2020).
25. Starr, T. N. et al. Deep mutational scanning of SARS-CoV-2 receptor-binding domain reveals constraints on folding and ACE2 binding. Cell. https://doi. org/10.1016/j.cell.2020.08.012 (2020).

26. $\mathrm{Wu}, \mathrm{K}$. et al. Serum neutralizing activity elicited by mRNA-1273 vaccine. N. Engl. J. Med. 384, 1468-1470 (2021).

27. Novavax. Novavax COVID-19 vaccine demonstrates $89.3 \%$ efficacy in UK phase 3 trial. Press Release 28 January 2021. https://ir.novavax.com/ node/15506/pdf (2021).

28. Emary, K. et al. Efficacy of ChAdOx1 nCoV-19 (AZD1222) vaccine against SARS-CoV-2 variant of concern 202012/01 (B.1.1.7): an exploratory analysis of a randomised controlled trial. Lancet https://doi.org/10.1016/S01406736(21)00628-0 (2021).

29. Sadoff, J. et al. Safety and efficacy of single-dose Ad26.COV2.S vaccine against COVID-19. N. Engl. J. Med. https://doi.org/10.1056/NEJMoa2101544 (2021).

30. Shinde, V. et al. Efficacy of NVX-CoV2373 COVID-19 vaccine against the B.1.351 variant. N. Engl. J. Med. 384, 1899-1909 (2021).

31. Abu-Raddad, L.J., Chemaitelly, H. \& Butt, A.A. Effectiveness of the BNT162b2 COVID-19 Vaccine against the B.1.1.7 and B.1.351 Variants. N. Engl. J. Med. 385, 187-189 (2021).

32. Madhi, S. A. et al. Efficacy of the ChAdOxl nCoV-19 COVID-19 vaccine against the B.1.351 variant. N. Engl. J. Med. 384, 1885-1898 (2021).

33. Corbett, K. S. et al. Evaluation of the mRNA-1273 vaccine against SARS-CoV-2 in nonhuman primates. N. Engl. J. Med. 383, 1544-1555 (2020).

34. Guebre-Xabier, M. et al. NVX-CoV2373 vaccine protects cynomolgus macaque upper and lower airways against SARS-CoV-2 challenge. Vaccine 38, 7892-7896 (2020).

35. Mercado, N. B. et al. Single-shot Ad26 vaccine protects against SARS-CoV-2 in rhesus macaques. Nature 586, 583-588 (2020).

36. van Doremalen, N. et al. ChAdOx1 nCoV-19 vaccination prevents SARS-CoV-2 pneumonia in rhesus macaques. Nature 586, 578-582 (2020).

37. Yu, J. et al. DNA vaccine protection against SARS-CoV-2 in rhesus macaques. Science 369, 806-811 (2020)

38. Corbett, K. S. et al. Immune correlates of protection by mRNA-1273 vaccine against SARS-CoV-2 in nonhuman primates. Science. https://doi.org/10.1126/ science.abj0299 (2021).

39. Klasse, P. J., Nixon, D. F. \& Moore, J. P. Immunogenicity of clinically relevant SARS-CoV-2 vaccines in nonhuman primates and humans. Sci. Adv. 7 , eabe8065 (2021).

40. Shen, X. et al. Neutralization of SARS-CoV-2 variants B.1.429 and B.1.351. N. Engl. J. Med. https://doi.org/10.1056/NEJMc2103740 (2021).

41. Pardi, N. et al. Nucleoside-modified mRNA vaccines induce potent $\mathrm{T}$ follicular helper and germinal center B cell responses. J. Exp. Med. 215 , 1571-1588 (2018).

42. Corbett, K. S. et al. SARS-CoV-2 mRNA vaccine development enabled by prototype pathogen preparedness. Nature 586, 567-571 (2020).

43. Zou, L. et al. SARS-CoV-2 viral load in upper respiratory specimens of infected patients. N. Engl. J. Med. 382, 1177-1179 (2020).

44. Wölfel, R. et al. Virological assessment of hospitalized patients with COVID-19. Nature 581, 465-469 (2020).

45. Francica, J. R. et al. Protective antibodies elicited by SARS-CoV-2 spike protein vaccination are boosted in the lung after challenge in nonhuman primates. Science Transl. Med. https://doi.org/10.1126/scitranslmed.abi4547 (2021).

46. Baden, L. R. et al. Efficacy and safety of the mRNA-1273 SARS-CoV-2 vaccine. N. Engl. J. Med. 384, 403-416 (2020).

47. Polack, F. P. et al. Safety and efficacy of the BNT162b2 mRNA COVID-19 vaccine. N. Engl. J. Med. 383, 2603-2615 (2020).

48. Shen, X. et al. SARS-CoV-2 variant B.1.1.7 is susceptible to neutralizing antibodies elicited by ancestral spike vaccines. Cell Host Microbe https://doi. org/10.1016/j.chom.2021.03.002 (2021).

49. Gao, Q. et al. Development of an inactivated vaccine candidate for SARS-CoV-2. Science 369, 77-81 (2020).

50. Pardi, N., Hogan, M. J., Porter, F. W. \& Weissman, D. mRNA vaccines-a new era in vaccinology. Nat. Rev. Drug Discov. 17, 261-279 (2018).

51. Munster, V. J. Subtle differences in the pathogenicity of SARS-CoV-2 variants of concern B.1.1.7 and B.1.351 in rhesus macaques. Preprint at https://doi. org/10.1101/2021.05.07.443115 (2021).

52. Radvak, P. B.1.1.7 and B.1.351 variants are highly virulent in K18-ACE2 transgenic mice and show different pathogenic patterns from early SARSCoV-2 strains. Preprint at https://doi.org/10.1101/2021.06.05.447221 (2021).

53. McMahan, K. et al. Correlates of protection against SARS-CoV-2 in rhesus macaques. Nature 590, 630-634 (2021).

Publisher's note Springer Nature remains neutral with regard to jurisdictional claims in published maps and institutional affiliations.

This is a U.S. government work and not under copyright protection in the U.S.; foreign copyright protection may apply 2021 


\section{Methods}

Propagation and characterization of viral stocks. VeroE6 cells were obtained from the American Type Culture Collection (ATCC; clone E6, CRL-1586). SARS-CoV-2/human/USA/GA-EHC-083E/2020 (referred to as the p.Asp614Gly variant) was derived from a residual nasopharyngeal swab collected from a patient of Emory Healthcare in March 2020. The isolation and sequencing were previously described $^{3}$. The B.1.351 variant isolate, kindly provided by A. Pekosz (JHU), was propagated once in Vero-TMPRSS2 cells (generated by VRC/National Institutes of Health (NIH)) to generate P2 viral stocks. Viral titers were determined by plaque assay on Vero-TMPRSS2 cells. Viral stocks were sequenced as described below and stored at $-80^{\circ} \mathrm{C}$ until use.

Deep sequencing of virus isolate. Illumina-ready libraries were generated using NEBNext Ultra II RNA Prep reagents (New England Biolabs) as previously described ${ }^{45}$. Briefly, we fragmented RNA, followed by double-stranded cDNA synthesis, end repair, and adaptor ligation. The ligated DNA was then barcoded and amplified by a limited cycle PCR and the barcoded Illumina libraries were sequenced by using a paired-end 150-bp protocol on a NextSeq 2000 (Illumina). Demultiplexed sequence reads were analyzed in the CLC Genomics Workbench v.21.0.3 by (1) trimming for quality, length and adaptor sequence; (2) mapping to the Wuhan-Hu-1 SARS-CoV-2 reference (GenBank accession no. NC_045512), (3) improving the mapping by local realignment in areas containing insertions and deletions (indels); and (4) generating both a sample consensus sequence and a list of variants. Default settings were used for all tools.

Titration of B.1.351 in golden Syrian hamsters. Hamster experiments were carried out in compliance with US NIH regulations and approval from the Animal Care and Use Committee of Bioqual. Challenge studies were conducted at Bioqual. Golden Syrian hamsters (Envigo, 089), aged 8- to 9-weeks old, were randomized into groups of ten based on weight, with each group containing a 1:1 male:female ratio. Hamsters were inoculated intranasally with a total dose of $1 \times 10^{3}-1 \times 10^{5}$ PFUs of SARS-CoV-2 JHU B.1.351 P2 in a final volume of $100 \mu$ split between each nostril. Body weight observations were made daily after infection.

Titration of B.1.351 in rhesus macaques. Three-year-old male and female rhesus macaques of Indian origin were sorted by age and weight and then stratified into groups. NHPs were challenged with total doses of $2.4 \times 10^{5}$ and $2.4 \times 10^{6}$ PFUs of JHU SARS-CoV-2 B.1.351 P2; the viral inoculum was administered in $3 \mathrm{ml}$ intratracheally and $1 \mathrm{ml}$ intranasally $(0.5 \mathrm{ml}$ into each nostril). On days 2,4 and 6 after challenge, BAL and NS samples were collected and assessed for sgRNA as detailed below.

Preclinical mRNA-1273 mRNA and lipid nanoparticle production process. A sequence-optimized mRNA encoding prefusion-stabilized SARS-CoV-2 $\mathrm{S}-2 \mathrm{P}^{54,55}$ protein was synthesized in vitro. The mRNA was purified by oligo-dT affinity purification and encapsulated in a lipid nanoparticle through a modified ethanol-drop nanoprecipitation process described previously ${ }^{56}$.

Rhesus macaque model. NHP experiments were carried out in compliance with US NIH regulations and approval from the Animal Care and Use Committee of the VRC and Bioqual. Challenge studies were conducted at Bioqual. Male and female, 3- to 12-year-old, Indian-origin rhesus macaques were sorted by sex, age and weight and then stratified into groups of eight animals. NHPs were immunized intramuscularly at week 0 and week $4-5$ with either $30 \mu \mathrm{g}$ or $100 \mu \mathrm{g}$ mRNA-1273 in $1 \mathrm{ml}$ of $1 \times$ PBS into the right hind leg or $30 \mu \mathrm{g}$ at week 0 . Naive aged-matched NHPs were included as controls. At week 12 (7-8 weeks after boost or 12 weeks after the single vaccination), all NHPs were challenged with a total dose of $5 \times 10^{5}$ PFUs of SARS-CoV-2 B.1.351 strain. The viral inoculum was administered as $3.75 \times 10^{5}$ PFUs in $3 \mathrm{ml}$ intratracheally and $1.25 \times 10^{5}$ PFUs in $1 \mathrm{ml}$ intranasally in a volume of $0.5 \mathrm{ml}$ into each nostril. Pre- and post-challenge sample collection is detailed in Extended Data Fig. 1.

Quantification of SARS-CoV-2 RNA and sgRNA. At the time of collection, NS samples were frozen in $1 \mathrm{ml}$ of $1 \times$ PBS containing $1 \mu \mathrm{l}$ of SUPERase-In RNase Inhibitor (Invitrogen) and BAL fluid was mixed with $1 \mathrm{ml}$ of RNAzol BD containing $10 \mu \mathrm{l}$ acetic acid and both were frozen at $-80^{\circ} \mathrm{C}$ until extraction. Nasal specimens were thawed at $55^{\circ} \mathrm{C}$, and the swab removed. The remaining PBS was mixed with $2 \mathrm{ml}$ of RNAzol BD (Molecular Research Center) and $20 \mu \mathrm{l}$ acetic acid. At the time of collection, $1 \mathrm{ml}$ of BAL fluid was mixed with $1 \mathrm{ml}$ of RNAzol BD containing $10 \mu \mathrm{l}$ acetic acid and frozen at $-80^{\circ} \mathrm{C}$ until extraction. BAL specimens were thawed at $20-22^{\circ} \mathrm{C}$ and mixed with an additional $1 \mathrm{ml}$ of RNAzol BD containing $10 \mu \mathrm{l}$ acetic acid. Total RNA was extracted from nasal specimens and BAL fluid using RNAzol BD Column Kits and eluted in $65 \mu \mathrm{l}$ water. Subgenomic SARS-CoV-2 E and N mRNA was quantified via RT-PCR as previously described ${ }^{38}$. Reactions were conducted with $5 \mu$ RNA and TaqMan Fast Virus 1-Step Master Mix (Applied Biosystems) with $500 \mathrm{nM}$ of primers and $200 \mathrm{nM}$ of probes. Primers and probes were as follows: sgLeadSARSCoV2_F: 5'-CGATCTCTTGTAGATCTGTTCTC-3', E subgenomic mRNA-E_Sarbeco_P: 5'-FAM-ACACTAGCCATCCTTACTGCGCTTCG-BHQ1-3' and E_Sarbeco_R:
5' -ATATTGCAGCAGTACGCACACA-3', N subgenomic mRNA-wtN_P: 5'-FAM-TAACCAGAATGGAGAACGCAGTGGG-BHQ1-3' and wtN_R 5'-GGTGAACCAAGACGCAGTAT-3'. Reactions were run on a QuantStudio 6 Pro Real-Time PCR System (Applied Biosystems) at the following conditions: $50^{\circ} \mathrm{C}$ for $5 \mathrm{~min}, 95^{\circ} \mathrm{C}$ for $20 \mathrm{~s}$, and $40 \mathrm{cycles}$ of $95^{\circ} \mathrm{C}$ for $15 \mathrm{~s}$ and $60^{\circ} \mathrm{C}$ for $1 \mathrm{~min}$. Absolute quantification was performed in comparison to a standard curve. For the standard curve, the $\mathrm{E}$ or $\mathrm{N}$ subgenomic mRNA sequence was inserted into a pcDNA3.1 vector (GenScript) and transcribed using MEGAscript T7 Transcription Kit (Invitrogen) followed by MEGAclear Transcription Clean-Up Kit (Invitrogen). The lower limit of quantification was 50 copies.

TCID $_{50}$ quantification of SARS-CoV-2 from BAL. Vero-TMPRSS2 cells (VRC/ $\mathrm{NIH}$ ) were plated at 25,000 cells per well in DMEM $+10 \% \mathrm{FBS}+$ gentamicin and the cultures were incubated at $37^{\circ} \mathrm{C}, 5.0 \% \mathrm{CO}_{2}$ until cells were $80-100 \%$ confluent the following day. Medium was aspirated and replaced with $180 \mu \mathrm{l}$ of DMEM $+2 \%$ FBS + gentamicin. Twenty microliters of BAL sample was added to the top row in quadruplicate and mixed using a P200 pipettor five times. Using the pipettor, $20 \mu \mathrm{l}$ was transferred to the next row, and repeated down the plate (columns A-H) representing tenfold dilutions. The tips were disposed for each row and repeated until the last row. Positive (virus stock of known infectious titer in the assay) and negative (medium only) control wells were included in each assay setup. The plates were incubated at $37^{\circ} \mathrm{C}, 5.0 \% \mathrm{CO}_{2}$ for $4 \mathrm{~d}$. The cell monolayers were visually inspected for cytopathic effect. TCID $_{50}$ values were calculated using the ReedMuench formula. For optimal assay performance, the $\mathrm{TCID}_{50}$ value of the positive control should test within twofold of the expected value.

Histopathology and immunohistochemistry. As previously described ${ }^{33}$, NHP lung tissue sections were stained with $\mathrm{H} \& \mathrm{E}$ for routine histopathology and a rabbit polyclonal SARS-CoV-2 (GeneTex, GTX135357) for detection of SARS-CoV-2 virus antigen. All samples were evaluated by a board-certified veterinary pathologist.

10-plex mesoscale ELISA. Multiplexed plates (96-well) precoated with SARS-CoV-2 S-2 $\mathrm{P}^{55}$ and RBD proteins from the following strains: WA-1, B.1.351, B.1.1.7, and P.1., SARS-CoV-2 N protein and BSA were supplied by the manufacturer (MSD). See Supplementary Table 3 for reagent details. Determination of antibody binding was performed as previously described ${ }^{20}$ Briefly, on the day of the assay, the plate was blocked for 60 min with MSD Blocker A (5\% BSA). The blocking solution was washed off and test samples were applied to the wells at four dilutions (1:100, 1:500, 1:2,500 and 1:10,000), unless otherwise specified and allowed to incubate with shaking for $2 \mathrm{~h}$. Plates were washed and SULFO-TAG-labeled anti-IgG antibody was applied to the wells and allowed to associate with complexed coated antigen-sample antibody within the assay wells. Plates are washed to remove unbound detection antibody. A read solution containing ECL substrate was applied to the wells, and the plate was entered into the MSD Sector instrument. A current was applied to the plate and areas of well surface where sample antibody was complexed with coated antigen and the labeled reporter emit light in the presence of the ECL substrate. The MSD Sector instrument quantified the amount of light emitted and reports this ECL unit response as a result for each sample and standard of the plate. The magnitude of ECL response is directly proportional to the extent of binding antibody in the test article. All calculations were performed in Excel and GraphPad Prism v7.0. Readouts are provided as AUC values.

4-plex mesoscale ELISA. MSD SECTOR plates were precoated by the manufacturer with SARS-CoV-2 proteins (S-2P $\mathrm{P}^{55}, \mathrm{RBD}$ and $\mathrm{N}$ ) and a BSA control in each well in a specific spot designation for each antigen. See Supplementary Table 3 for reagent details. Determination of antibody binding was performed as previously described ${ }^{38}$. In detail, serum samples were heat inactivated for $30 \mathrm{~min}$ at $56^{\circ} \mathrm{C}$ before assay. Plates were blocked for $60 \mathrm{~min}$ at room temperature (RT) with MSD blocker A solution without shaking. Plates were washed and the MSD reference standard (calibrator), quality-control test sample (pool of COVID-19 convalescent sera) and human serum test samples were added to the precoated wells in duplicates in an eight-point dilution series. Reference standard was added in triplicates. MSD control sera (low, medium and high) were added undiluted in triplicates per validated assay format. Additional assay controls can be added in triplicates. Samples were incubated at RT for $4 \mathrm{~h}$ with shaking on a Titramax Plate shaker (Heidolph) at 1,500 r.p.m. SARS-CoV-2-specific antibodies present in the sera or controls bind to the coated antigens. Plates were washed to remove unbound antibodies. Antibodies bound to the SARS-CoV-2 viral proteins were detected using an MSD SULFO-TAG anti-human IgG detection antibody incubated for $60 \mathrm{~min}$ at RT and with shaking. Plates were washed and a read solution (MSD GOLD read buffer) containing electrochemiluminescence (ECL) substrate was applied to the wells and the plate was entered into the MSD MESO Sector S 600 detection system. An electric current was applied to the plates and areas of well surface that form antigen anti-human IgG antibody SULFO-TAG complex emit light in the presence of the ECL substrate.

The MSD MESO Sector S 600 detection system quantifies the amount of light emitted and reports the ECL unit response as a result for each test sample, 
control sample and reference standard of each plate. Analysis was performed with the MSD Discovery Workbench software, version 4.0. Calculated ECL immunoassay parameters to measure binding antibody activities include interpolated concentrations or assigned arbitrary units (a.u. per ml) read from the standard curve.

Conversion to 4-plex readout to international units. Recently the arbitrary units were bridged to the WHO International Standard (provided in international units per $\mathrm{ml}\left(\mathrm{IU} \mathrm{ml} \mathrm{ml}^{-1}\right)$ ), and a conversion factor was calculated and confirmed. Parallelism was established for all three antigens (SARS-CoV S-2P, RBD and $\mathrm{N}$ ) between the MSD-provided reference standard and the WHO-provided international standard. Concentration assignments were performed and then confirmed both at MSD and as part of a multisite confirmation study.

Mesoscale ELISA for mucosal antibody responses. Using previously described methods ${ }^{45}$, total S-specific IgG and IgA were determined by MULTI-ARRAY ELISA using MSD.

Lentiviral pseudovirus neutralization assay. As previously described ${ }^{57}$, pseudotyped lentiviral reporter viruses were produced by the co-transfection of plasmids encoding S proteins from Wuhan-1 strain (Genbank no. MN908947.3) with a p.Asp614Gly mutation, a luciferase reporter, lentivirus backbone and human transmembrane protease serine 2 (TMPRSS2) genes into HEK293T/17 cells (ATCC CRL-11268). Similarly, pseudoviruses containing S from B.1.351, P.1 and B.1.1.7 were produced. See Supplementary Table 3 for reagent details. Sera, in duplicate, were tested for neutralizing activity against the pseudoviruses by quantification of luciferase activity (in relative light units). The percentage of neutralization was normalized considering uninfected cells as $100 \%$ neutralization and cells infected with pseudovirus alone as $0 \%$ neutralization. $\mathrm{IC}_{50}$ titers were determined using a $\log$ (agonist) versus normalized-response (variable slope) nonlinear regression model in Prism v9.0.2 (GraphPad). For samples that did not neutralize at the limit of detection at $50 \%$, a value of 20 was plotted and used for geometric mean calculations.

VSV pseudovirus neutralization assay. To make SARS-CoV-2 pseudotyped recombinant VSV- $\Delta$ G-firefly luciferase virus, BHK21/WI-2 cells (Kerafast, EH1011) were transfected with the Wuhan-1 strain (Genbank no. MN908947.3) S plasmid expressing full-length S with p.Asp614Gly mutation or S of B.1.351. See Supplementary Table 3 for reagent details. After S plasmid transfection, cells were subsequently infected with VSV $\Delta \mathrm{G}$-firefly-luciferase as previously described ${ }^{58}$. Neutralization assays were completed on A549-ACE2-TMPRSS2 cells, generated using lentivirus encoding hACE2-P2A-TMPRSS2, with serially diluted serum samples as previously described ${ }^{26}$. For samples that did not neutralize at the limit of detection at $50 \%$, a value of 20 was plotted and used for geometric mean calculations.

Focus-reduction neutralization test. Viruses were propagated in Vero-TMPRSS2 cells (VRC/NIH) (to generate viral stocks. See Supplementary Table 3 for reagent details. Viral titers were determined by focus-forming assay on VeroE6 cells (ATCC). Viral stocks were stored at $-80^{\circ} \mathrm{C}$ until use. Focus-reduction neutralization test assays were performed as previously described ${ }^{59}$. In detail, samples were diluted at threefold in eight serial dilutions using DMEM (VWR, 45000-304) in duplicates with an initial dilution of 1:10 in a total volume of $60 \mathrm{ml}$. Serially diluted samples were incubated with an equal volume of SARS-CoV-2 (100-200 foci per well) at $37^{\circ} \mathrm{C}$ for $1 \mathrm{~h}$ in a round-bottomed 96 -well culture plate. The antibody-virus mixture was then added to Vero cells and incubated at $37^{\circ} \mathrm{C}$ for $1 \mathrm{~h}$. After incubation, the antibody-virus mixture was removed and $100 \mathrm{\mu l}$ of prewarmed $0.85 \%$ methylcellulose (Sigma-Aldrich, M0512-250G) overlay was added to each well. Plates were incubated at $37^{\circ} \mathrm{C}$ for $24 \mathrm{~h}$. After $24 \mathrm{~h}$, methylcellulose overlay was removed, and cells were washed three times with PBS. Cells were then fixed with $2 \%$ paraformaldehyde in PBS (Electron Microscopy Sciences) for $30 \mathrm{~min}$. Following fixation, plates were washed twice with PBS and $100 \mu \mathrm{l}$ of permeabilization buffer (0.1\% BSA (VWR, 0332), saponin (Sigma, 47036-250G-F) in PBS) was added to the fixed Vero cells for $20 \mathrm{~min}$. Cells were incubated with an anti-SARS-CoV S primary antibody directly conjugated to biotin (CR3022-biotin) for $1 \mathrm{~h}$ at RT. Next, the cells were washed $3 \times$ in PBS and avidin-HRP was added for $1 \mathrm{~h}$ at RT followed by three washes in PBS. Foci were visualized using TrueBlue HRP substrate (KPL, 5510-0050) and imaged on an ELISPOT reader (CTL). Antibody neutralization was quantified by counting the number of foci for each sample using the Viridot program ${ }^{60}$. The neutralization titers were calculated as follows: 1 - (ratio of the mean number of foci in the presence of sera and foci at the highest dilution of the respective sera sample). Each specimen was tested in duplicate. The $50 \%$ focus-reduction neutralization test titers were interpolated using a four-parameter nonlinear regression in GraphPad Prism v9.0.2.4.3. For samples that did not neutralize at the limit of detection at $50 \%$, a value of 5 was plotted and used for geometric mean calculations.

Intracellular cytokine staining. Cryopreserved peripheral blood mononucleolar cells were thawed and rested overnight in a $37^{\circ} \mathrm{C} / 5 \% \mathrm{CO}_{2}$ incubator. The next morning, cells were stimulated with SARS-CoV-2 S-protein peptide pools (S1 and S2) that were matched to the vaccine insert (comprising 158 and 157 individual peptides, respectively, as 15 -mers overlapping by 11 amino acids in $100 \%$ dimethyl sulfoxide; JPT Peptides) at a final concentration of $2 \mu \mathrm{g} \mathrm{ml}^{-1}$ in the presence of $3 \mu \mathrm{M}$ monensin for $6 \mathrm{~h}$. Negative controls received an equal concentration of dimethyl sulfoxide instead of peptides (final concentration of $0.5 \%$ ). Intracellular cytokine staining was performed as described previously ${ }^{61,62}$. The following monoclonal antibodies were used: CD3 APC-Cy7 (clone SP34.2, BD Biosciences), CD4 PE-Cy5.5 (clone S3.5, Invitrogen), CD8 BV570 (clone RPA-T8, BioLegend), CD45RA PE-Cy5 (clone 5H9, BD Biosciences), CCR7 BV650 (clone G043H7, BioLegend), CXCR5 PE (clone MU5UBEE, Thermo Fisher), CXCR3 BV711 (clone 1C6/CXCR3, BD Biosciences), PD-1 BUV737 (clone EH12.1, BD Biosciences), ICOS Pe-Cy7 (clone C398.4 A, BioLegend), CD69 ECD (cloneTP1.55.3, Beckman Coulter), IFN- $\gamma$ Ax700 (clone B27, BioLegend), IL-2 BV750 (clone MQ1-17H12, BD Biosciences), IL-4 BB700 (clone MP4-25D2, BD Biosciences), TNF-FITC (clone Mab11, BD Biosciences), IL-13 BV421 (clone JES10-5A2, BD Biosciences), IL-17 BV605 (clone BL168, BioLegend), IL-21 Ax647 (clone 3A3-N2.1, BD Biosciences) and CD154 BV785 (clone 24-31, BioLegend). Aqua live/dead fixable dead cell stain kit (Thermo Fisher Scientific) was used to exclude dead cells. All antibodies were previously titrated to determine the optimal concentration. Samples were acquired on an BD FACSymphony flow cytometer and analyzed using FlowJo version 9.9.6 (Treestar) following the gating scheme in Extended Data Fig. 7.

Statistical analysis. Graphs show data from individual NHPs with dotted lines indicating assay limits of detection. Groups were compared with the KruskalWallis test, followed by pairwise two-sided Wilcoxon rank-sum tests with Holm's adjustment on the set of pairwise tests if the Kruskal-Wallis test was significant, for the primary analysis of viral load at day 2 in the BAL and NS samples, as well as other comparisons between dose groups. Correlations were estimated and tested using Spearman's nonparametric method. Linear regression was used to explore the relationship between antibody levels and sgRNA, including quadratic terms for comparing the newly generated data and those previously published ${ }^{61}$, with likelihood-ratio tests to compare models and assess interaction effects.

Reporting Summary. Further information on research design is available in the Nature Research Reporting Summary linked to this article.

\section{Data availability}

The datasets generated during and/or analyzed during the current study are available from the corresponding author on reasonable request. Source data are provided with this paper.

\section{References}

54. Pallesen, J. et al. Immunogenicity and structures of a rationally designed prefusion MERS-CoV spike antigen. Proc. Natl Acad. Sci. USA 114, E7348-E7357 (2017)

55. Wrapp, D. et al. Cryo-EM structure of the 2019-nCoV spike in the prefusion conformation. Science 367, 1260-1263 (2020).

56. Hassett, K. J. et al. Optimization of lipid nanoparticles for intramuscular administration of mRNA vaccines. Mol. Ther. Nucleic Acids 15, 1-11 (2019).

57. Jackson, L. A. et al. An mRNA vaccine against SARS-CoV-2-preliminary report. N. Engl. J. Med. 383, 1920-1931 (2020).

58. Whitt, M. A. Generation of VSV pseudotypes using recombinant $\Delta \mathrm{G}-\mathrm{VSV}$ for studies on virus entry, identification of entry inhibitors, and immune responses to vaccines. J. Virol. Methods 169, 365-374 (2010).

59. Vanderheiden, A. et al. Development of a rapid focus-reduction neutralization test assay for measuring SARS-CoV-2 neutralizing antibodies. Curr. Protoc. Immunol. 131, e116 (2020).

60. Katzelnick, L. C. et al. Viridot: an automated virus plaque (immunofocus) counter for the measurement of serological neutralizing responses with application to dengue virus. PLoS Negl. Trop. Dis. 12, e0006862 (2018).

61. Donaldson, M. M., Kao, S. F. \& Foulds, K. E. OMIP-052: an 18-color panel for measuring $\mathrm{T}_{\mathrm{H}} 1, \mathrm{~T}_{\mathrm{H}} 2, \mathrm{~T}_{\mathrm{H}} 17$ and $\mathrm{T}_{\mathrm{FH}}$ responses in rhesus macaques. Cytometry A 95, 261-263 (2019).

62. Finak, G. et al. Mixture models for single-cell assays with applications to vaccine studies. Biostatistics 15, 87-101 (2013).

\section{Acknowledgements}

We thank any additional members of all included laboratories for critical discussions and advice pertaining to experiments included in the manuscript. We thank J. Stein and M. Young for technology transfer and administrative support, respectively. We thank members of the NIH NIAID VRC Translational Research Program, including C. Case, H. Bao, E. McCarthy, J. Noor, A. Taylor, and R. Woodward, for technical and administrative assistance with animal experiments. We thank H. Mu and M. Farzan, from The Scripps Research Institute, for the ACE2-overexpressing HEK293 cells. We thank A. Creanga and M. Kanekiyo for the Vero-TMPRSS2 cells. We thank the 
laboratory of P. Kwong for providing protein for use in ELISA assays for detection of mucosal antibodies. We thank M. Brunner and M. Whitt, from the University of Tennessee Health Science Center College of Medicine, for kind support on recombinant VSV-based SARS-CoV-2 pseudovirus production. This work was supported by the Intramural Research Program of the VRC, NIAID and NIH. mRNA-1273 has been funded in part with Federal funds from the Department of Health and Human Services, Office of the Assistant Secretary for Preparedness and Response, Biomedical Advanced Research and Development Authority, under contract 75A50120C00034. K.S.C.'s research fellowship was partially funded by the Undergraduate Scholarship Program, Office of Intramural Training and Education, Office of the Director, NIH. Virus propagation and live-virus neutralization assays were funded by Emory Executive Vice President for Health Affairs Synergy Fund Award, Pediatric Research Alliance Center for Childhood Infections and Vaccines and Children's Healthcare of Atlanta, Woodruff Health Sciences Center 2020 COVID-19 CURE Award and NIAID Centers of Excellence for Influenza Research and Surveillance (CEIRS) contract HHSN272201400004C (M.S.S.). M.S.S. is on the Advisory Board of Moderna.

\section{Author contributions}

K.S.C., A.P.W., S.O., M.G., L.L., J.I.M., B.F., A.C., M.K, K.E.F., S.F.A., D.R.F., E.L., S.T.N., S.J.P., K.W.B., M.M., B.M.N., A.V.R., Z.F., T.S.J., E.B.M., P.M., A.R.H., F.L., B.C., M.P., J.W. J.-P.M.T., B.B., A.C., A.D., L.P., K.S., S.E., H.A., K.W., D.K.E., S.K., M.G.L., E.B., I.N.M., A.C., M.S.S., A.M., M.R., M.C.N., N.J.S., D.C.D., B.S.G. and R.A.S. designed, completed and/or analyzed experiments. S.B.-B. and A.P. provided critical published reagents/ analytic tools. K.S.C., M.C.N. and R.A.S. wrote the manuscript. K.S.C., G.A., M.S. and
M.C.N. prepared figures and tables. All authors contributed to discussions in regard to and editing of the manuscript.

\section{Competing interests}

K.S.C. and B.S.G. are inventors on US Patent no. 10,960,070 B2 and International Patent Application no. WO/2018/081318 entitled 'Prefusion coronavirus spike proteins and their use.' K.S.C. and B.S.G. are inventors on US Patent Application no. 62/972,886 entitled '2019-nCoV vaccine'. A.C., M.K., S.E., K.W., D.K.E. and A.C. are employees of Moderna. A.V.R., Z.F., B.C., M.P., J.W., B.B., A.C., A.D., L.P., K.S., H.A., S.K. and M.G.L. are employees of Bioqual. M.S.S. serves on the scientific board of advisors for Moderna. The other authors declare no competing interests.

\section{Additional information}

Extended data is available for this paper at https://doi.org/10.1038/s41590-021-01021-0.

Supplementary information The online version contains supplementary material available at https://doi.org/10.1038/s41590-021-01021-0.

Correspondence and requests for materials should be addressed to B.S.G. or R.A.S

Peer review information Nature Immunology thanks the anonymous reviewers for their contribution to the peer review of this work. Editor recognition statement: Z. Fehervari was the primary editor on this article and managed its editorial process and peer review in collaboration with the rest of the editorial team.

Reprints and permissions information is available at www.nature.com/reprints. 


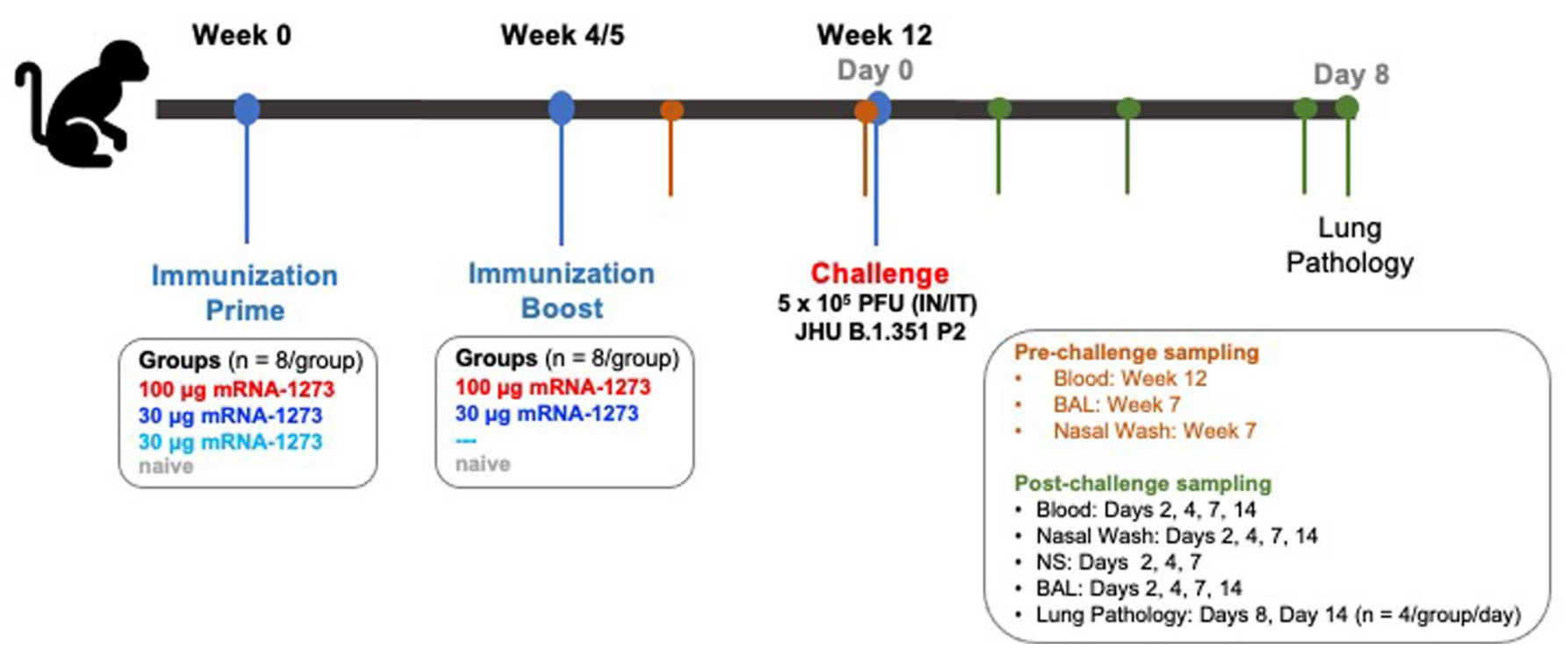

Extended Data Fig. 1 | Study design: Ability of mRNA-1273 to protect NHP against B.1.351 challenge. Nonhuman primates (NHP), Rhesus macaques $(n=8 /$ group), were immunized with mRNA-1273 on the following schedule: Group 1: 0 and 4 weeks, $100 \mu$; Group 2: 0 and 5 weeks, $30 \mu g ;$ Group 3: week $0,30 \mu \mathrm{g}$. Naive aged-matched NHP were included as controls. At week 12, animals were challenged with a total of $5 \times 10^{5}$ PFU of SARS-CoV-2 B.1.351. The viral inoculum was administered as $3.75 \times 10^{5} \mathrm{PFU}$ in $3 \mathrm{~mL}$ intratracheally (IT) and $1.25 \times 10^{5} \mathrm{PFU}$ in $1 \mathrm{~mL}$ intranasally (IN) in a volume of $0.5 \mathrm{~mL}$ into each nostril. Sera were collected at weeks 7 and week 12. Bronchoalveolar lavages (BAL) and nasal washes were also collected at week 7 . Sera, BAL, and nasal washes were collected post-challenge on days $2,4,7$, and 14 , as indicated. Lung pathology was assessed on day 8 post-challenge in a subset of animals ( $n=4$ /group). 
A

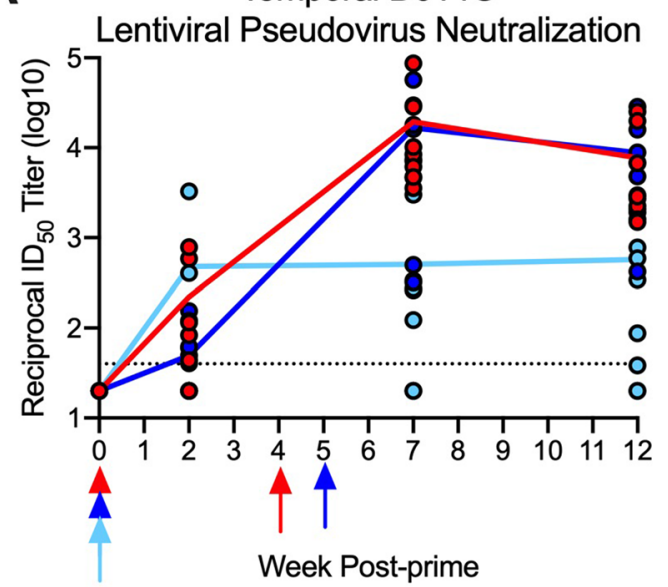

B Temporal B.1.351

Lentiviral Pseudovirus Neutralization

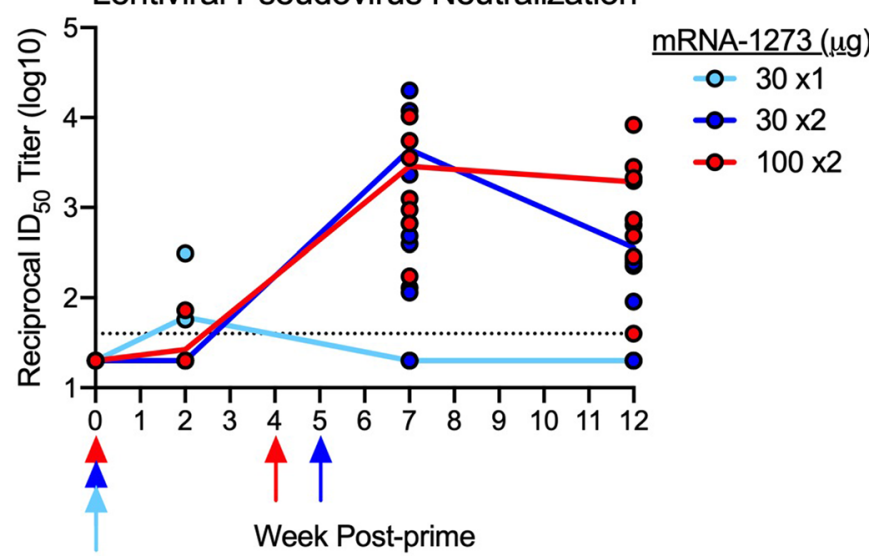

Extended Data Fig. 2 | Temporal neutralizing antibody responses following mRNA-1273 immunization. Rhesus macaques were immunized according to Supplementary Fig. 1. Sera collected at weeks 0, 2, 7, and 12 were assessed for SARS-CoV-2 D614G (A), and B.1.351 (B) lentiviral-based pseudovirus neutralization. Data represents one independent experiment. Circles represent individual NHP and may overlap where values are equal; lines represent geometric mean titers (GMT). Dotted lines indicate neutralization assay limits of detection. Arrows point to immunization weeks. 


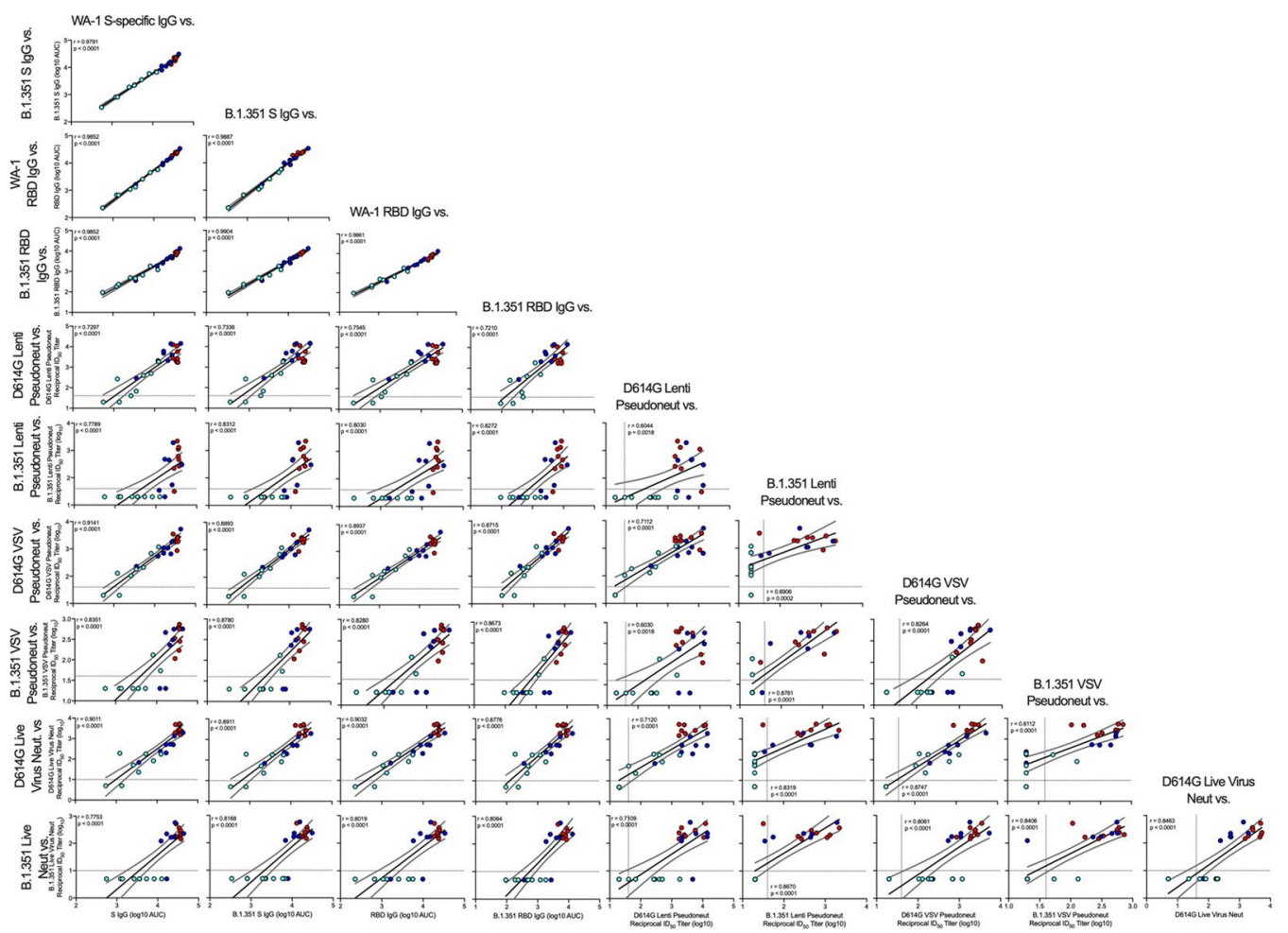

Extended Data Fig. 3 | Correlations of humoral antibody analyses. Rhesus macaques were immunized according to Supplementary Fig. 1c. Plots show correlations between SARS-CoV-2 WA-1 S-specific lgG, B.1.351 S-specific IgG, WA-1 RBD-specific lgG, B.1.351 RBD-specific lgG, D614G lentiviral-based pseudovirus neutralization, B.1.351 lentiviral-based pseudovirus neutralization, D614G VSV-based pseudovirus neutralization, B.1.351 VSV-based pseudovirus neutralization, D614G focus reduction neutralization, and B.1.351 focus reduction neutralization at week 12. Data represents one independent experiment. Circles represent individual NHP, where colors indicate mRNA-1273 dose as defined in Supplementary Fig. 1. Dotted lines indicate assay limits of detection. Black and gray lines indicate linear regression and $95 \%$ confidence interval, respectively. ' $r$ ' and ' $p$ ' represent Spearman's correlation coefficients and corresponding two-sided p-values, respectively. 
A

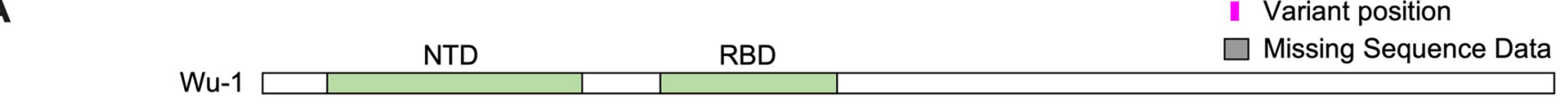

B.1.351 (Pango) ए ए

B.1.351 (JHU) ए ए

B.1.351 (JHU) P1 ए ए

B.1.351 (JHU) P2

\begin{tabular}{|c|c|c|c|c|c|}
\hline \begin{tabular}{|l|l|} 
& \\
\end{tabular} & 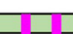 & $1 c_{0}$ & 7 & $\mathrm{C}$ & 7 \\
\hline 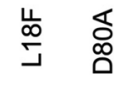 & 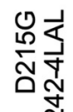 & $\frac{Z}{\frac{Z}{ \pm}}$ & 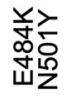 & O & \\
\hline
\end{tabular}

B Syrian Hamsters JHU B.1.351 P2 Viral Stock Titration Weight Loss

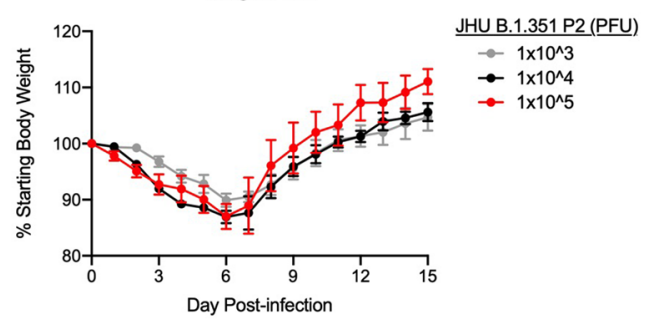

C

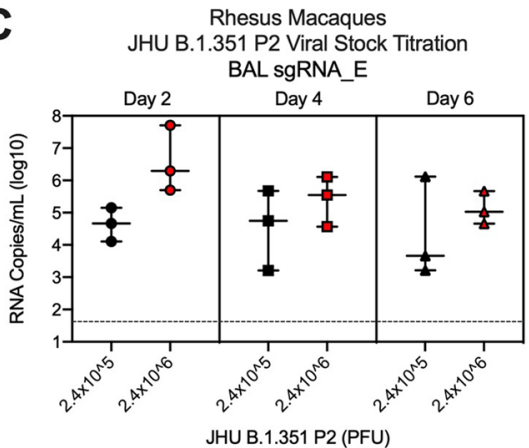

D

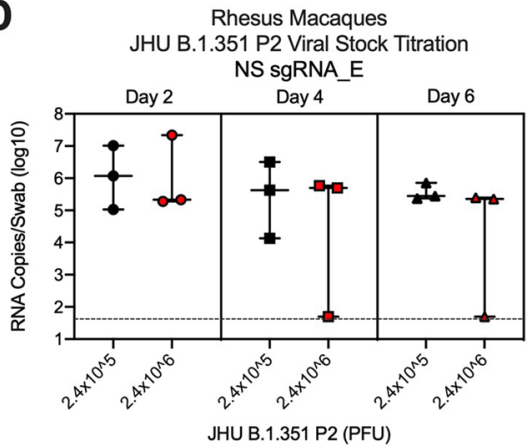

Extended Data Fig. 4 | Characterization of B.1.351 viral isolate. A SARS-CoV-2 B.1.351 clinical isolate was first passaged (P1) at Johns Hopkins University (JHU) on Vero cells then passaged again (P2) on Vero/TMPRSS2 cells. P1 and P2 underwent shotgun deep sequencing. (A) Alignment of S protein consensus sequence, where pink and gray indicate variant amino acid position and missing sequence data, respectively. (B) Syrian hamsters ( $n=10 /$ group) were infected with $1 \times 10^{3}$ (gray), $1 \times 10^{4}$ (black), or $1 \times 10^{5}$ (red) dilution of JHU B.1.351 P2 and monitored for weight loss for 15 days post-infection. Circles and error bars represent means and SEM, respectively. (C-D) Rhesus macaques ( $n=3$ /group) were infected with $2.4 \times 10^{5}$ (black) or $2.4 \times 10^{6}$ (red) PFU of JHU B.1.351 P2 and viral replication was assessed by detection of SARS-CoV-2 E-specific sgRNA in BAL (C) and NS (D) on days 2, 4, and 6 post-infection. Data represents one independent experiment. Boxes and horizontal bars denote the interquartile ranges (IQR) and medians, respectively; whisker end points are equal to the maximum and minimum values. Dotted lines indicate assay limits of detection. 
A

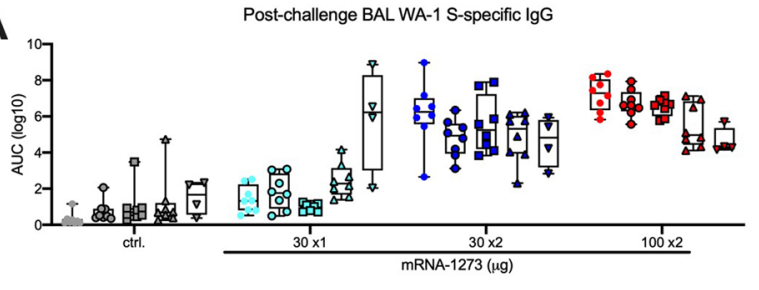

C

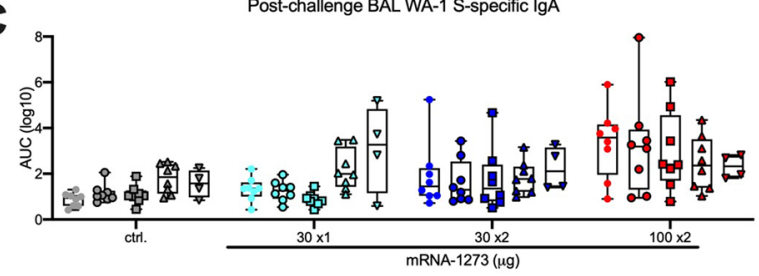

B

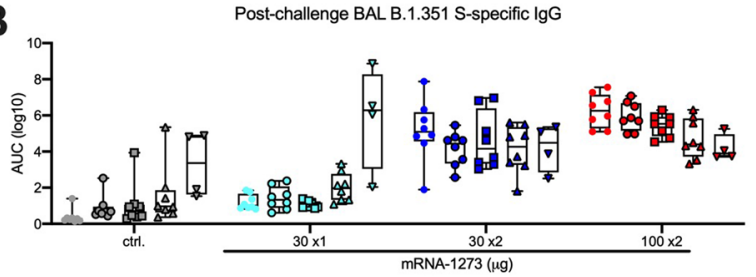

D

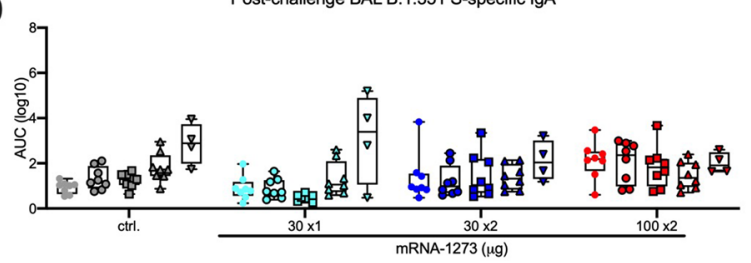

pre-challenge

ODay 2

Day 4

$\triangle$ Day 7

$\nabla$ Day 14
E

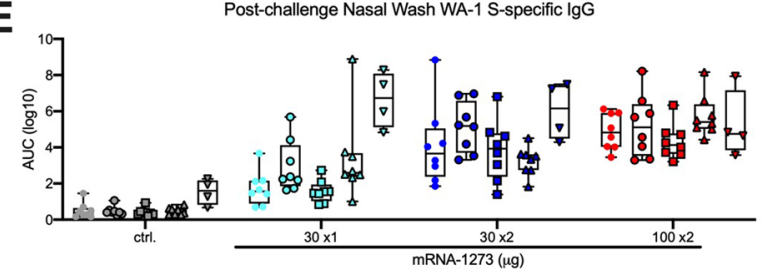

G

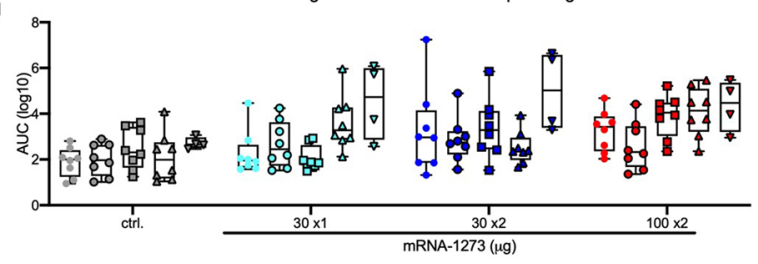

F

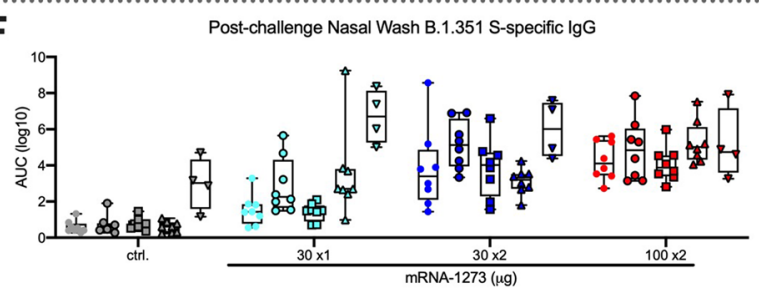

H

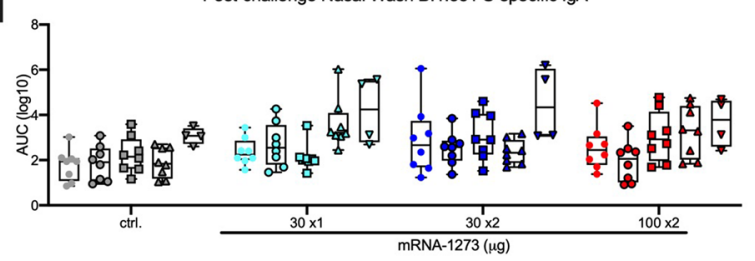

Extended Data Fig. 5 | Mucosal antibody responses following SARS-CoV-2 challenge in mRNA-1273-immunized NHP. Rhesus macaques were immunized and challenged according to Supplementary Fig. 1. BAL (A-D) and nasal washes (E-H) collected at week 7 (filled circles) and days 2 (circles), 4 (squares), 7 (triangles), and 14 (inverted triangles) post-challenge were assessed for SARS-CoV-2 WA-1 (A, C, E, G) and B.1.351 (B, D, F, H) S-specific $\operatorname{IgG}(A-B, E-F)$ and IgA (C-D, G-H) by MULTI-ARRAY ELISA. Data represents one independent experiment. Boxes and horizontal bars denote the IQR and medians, respectively; whisker end points are equal to the maximum and minimum values. Symbols represent individual NHP. 
A $\quad$ BAL CD4 - Th1 - S1+S2

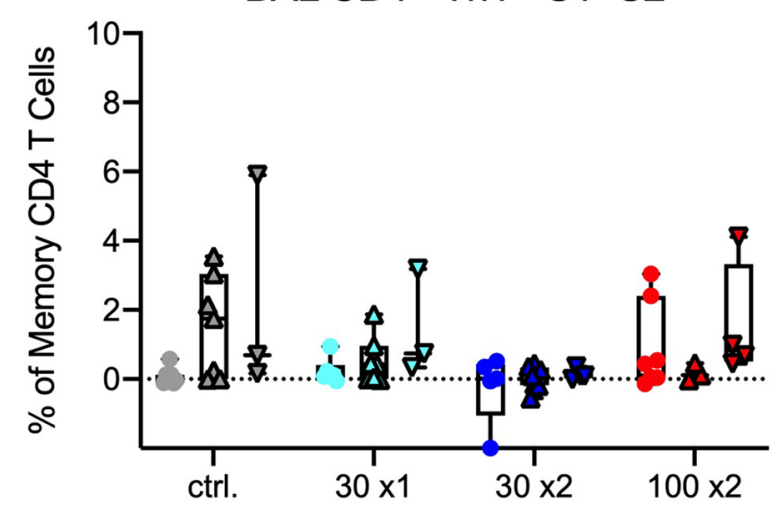

C

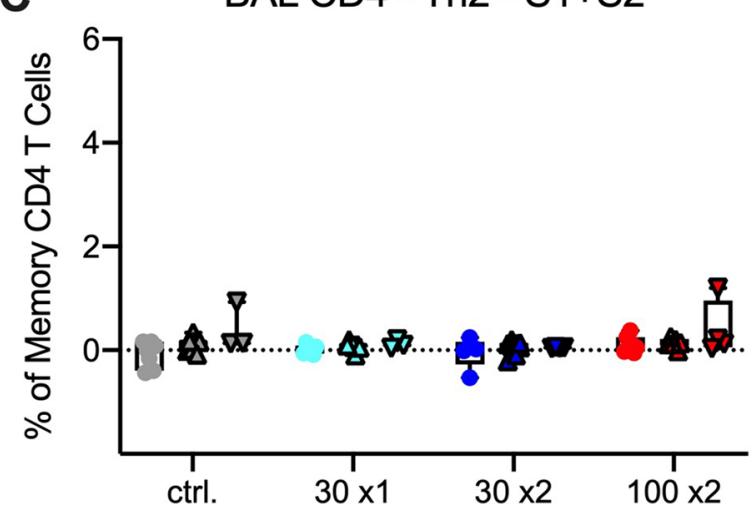

E

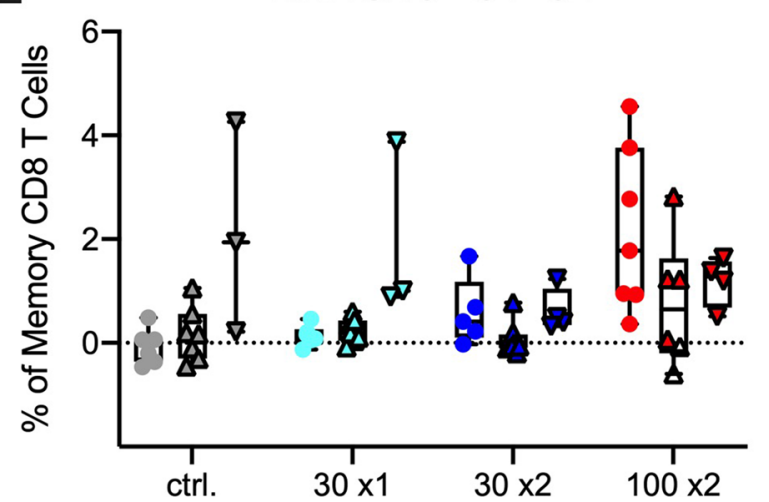

B

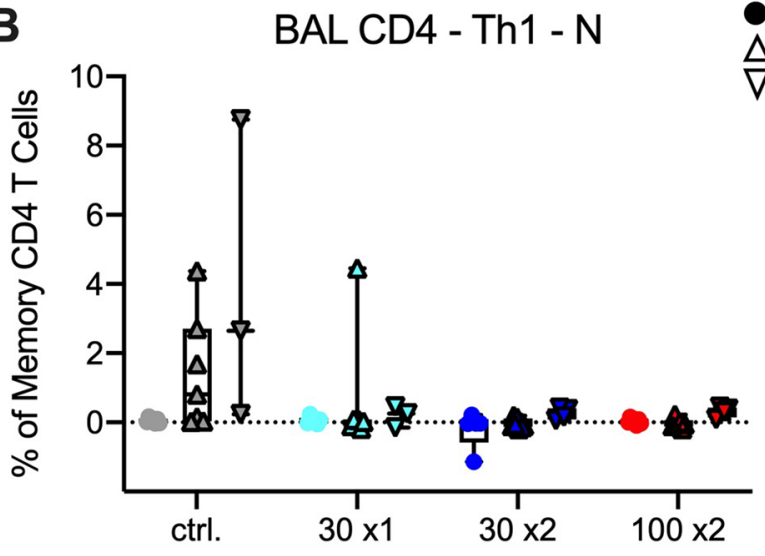

D

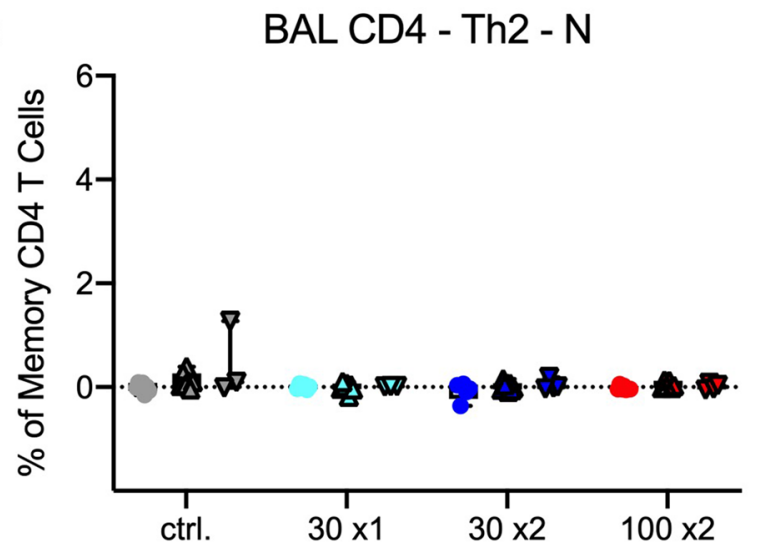

$\mathbf{F}$

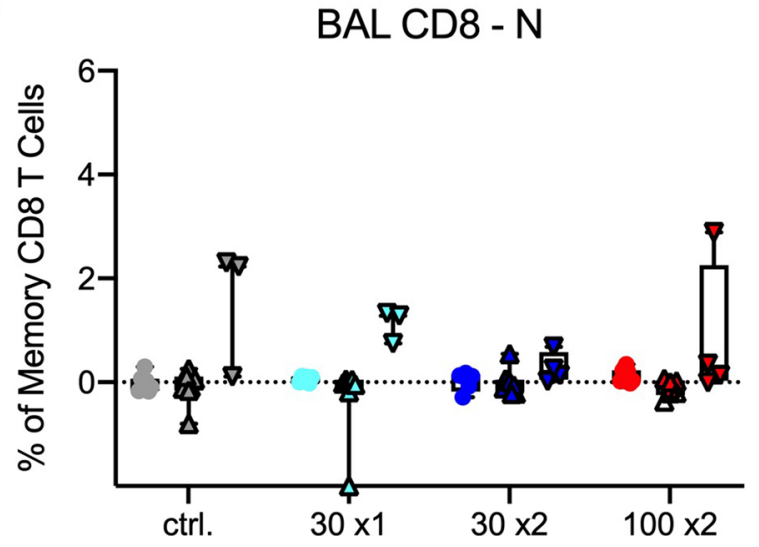

Extended Data Fig. 6 | T cell responses following SARS-CoV-2 challenge in mRNA-1273-immunized. Rhesus macaques were immunized according to Extended Data Fig. 1. Intracellular cytokine staining was performed on BAL cells at week 27 (filled circles) and days 7 (triangles) and 14 (inverted triangles) post-challenge to assess T cell responses to SARS-CoV-2 S protein peptide pools, S1 and S2 (A, C, E) and N (B, D, F). Responses to S1 and S2 individual peptide pools were summed. Cytokine frequencies were measured from memory $T$ cells as defined by CD45RA and CCR7. (A-B) Th1 responses (IFNg, IL-2, or TNF), (C-D) Th2 responses (IL-4 or IL-13), (E-F) CD8 T cell responses (IFNg, IL-2, or TNF). Data represents one independent experiment. Boxes and horizontal bars denote IQR and medians, respectively; whisker end points are equal to the maximum and minimum values. Circles represent individual NHP. Dotted lines are set to $0 \%$. 


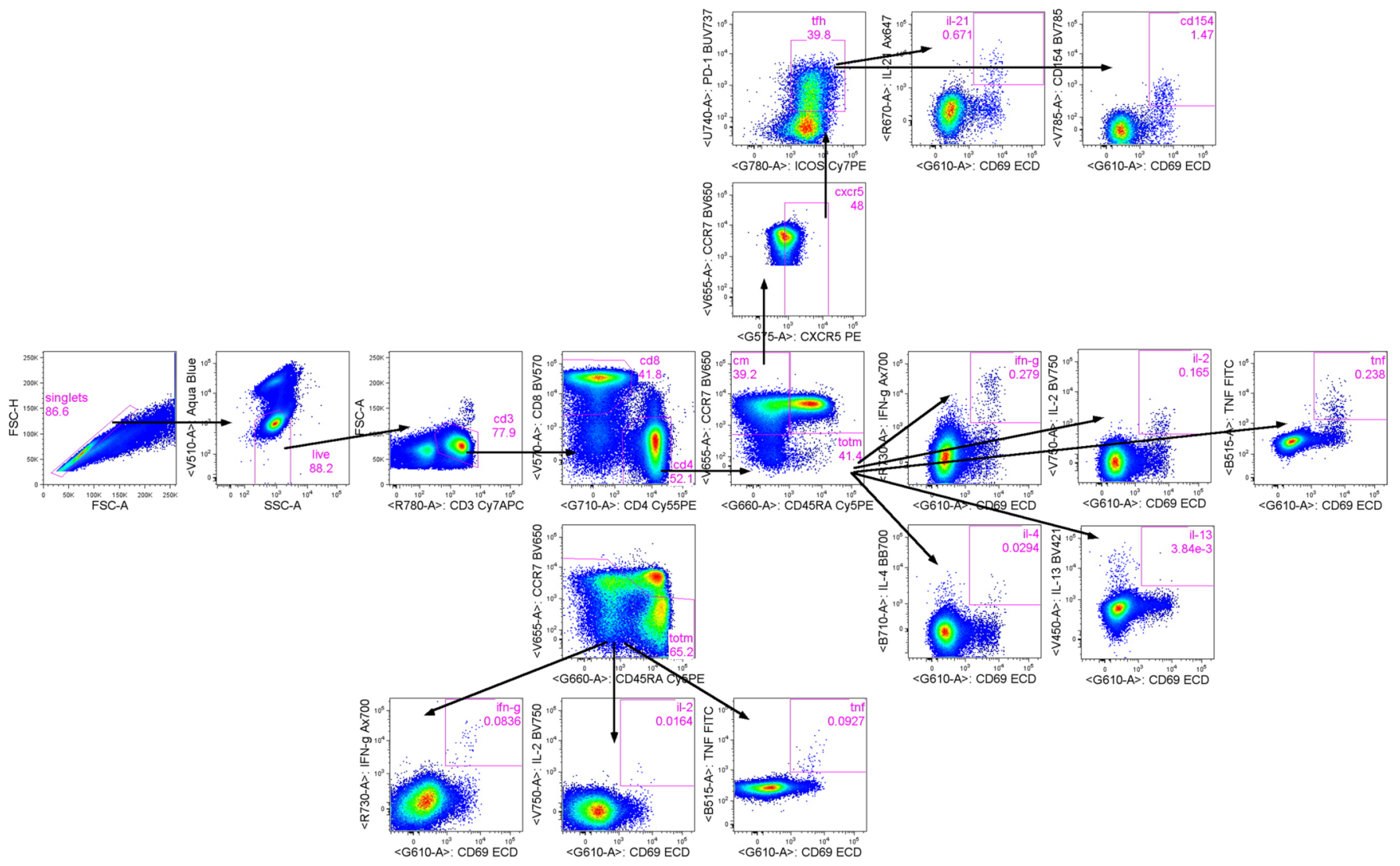

Extended Data Fig. 7 | Flow cytometry gating tree. T cell populations were selected by subsequent gating: first single cells, followed by live/SSC low cells, then CD3+ /FSC low cells, and finally CD4+ and CD8+ T cells. To measure IL-21 production and CD4OL expression from Tfh, CD4 T cells were gated first on central memory (CM) T cells (CCR7 + CD45RA-) then CXCR5 + CM cells followed by PD-1+ICOS + cells to identify Tfh. Subsequently, from Tfh, CD69 + IL-21+ and CD69+CD154 + cells were gated. Cytokine production from total memory (TotM) CD4 and CD8 T cells was measured by first gating on total memory cells (central memory CCR7 + CD45RA- plus effector memory CCR7-CD45RA- plus terminal effector memory CCR7-CD45RA + T cells) while excluding naive CCR7 + CD45RA + T cells, then CD69+ cytokine+ gates were drawn on total memory T cells. 


\section{Reporting Summary}

Nature Portfolio wishes to improve the reproducibility of the work that we publish. This form provides structure for consistency and transparency in reporting. For further information on Nature Portfolio policies, see our Editorial Policies and the Editorial Policy Checklist.

\section{Statistics}

For all statistical analyses, confirm that the following items are present in the figure legend, table legend, main text, or Methods section.

$\mathrm{n} / \mathrm{a} \mid$ Confirmed

$\bigotimes$ The exact sample size $(n)$ for each experimental group/condition, given as a discrete number and unit of measurement

\ A statement on whether measurements were taken from distinct samples or whether the same sample was measured repeatedly

The statistical test(s) used AND whether they are one- or two-sided

Only common tests should be described solely by name; describe more complex techniques in the Methods section.

Х A description of all covariates tested

\A description of any assumptions or corrections, such as tests of normality and adjustment for multiple comparisons

A full description of the statistical parameters including central tendency (e.g. means) or other basic estimates (e.g. regression coefficient)

AND variation (e.g. standard deviation) or associated estimates of uncertainty (e.g. confidence intervals)

For null hypothesis testing, the test statistic (e.g. $F, t, r$ ) with confidence intervals, effect sizes, degrees of freedom and $P$ value noted Give $P$ values as exact values whenever suitable.

Х $\square$ For Bayesian analysis, information on the choice of priors and Markov chain Monte Carlo settings

Х $\square$ For hierarchical and complex designs, identification of the appropriate level for tests and full reporting of outcomes

$\square \bigotimes$ Estimates of effect sizes (e.g. Cohen's d, Pearson's $r$ ), indicating how they were calculated

\section{Our web collection on statistics for biologists contains articles on many of the points above.}

\section{Software and code}

Policy information about availability of computer code

Data collection No data collection software was used.

Data analysis Prism v7.0, Prism v9.0.2, Prism v9.0.2.4.3, and MSD Discovery Workbench software, Version 4.0

For manuscripts utilizing custom algorithms or software that are central to the research but not yet described in published literature, software must be made available to editors and

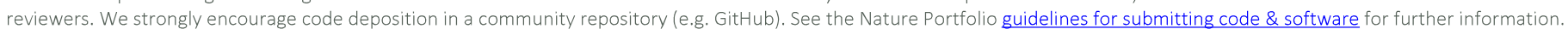

\section{Data}

Policy information about availability of data

All manuscripts must include a data availability statement. This statement should provide the following information, where applicable:

- Accession codes, unique identifiers, or web links for publicly available datasets

- A description of any restrictions on data availability

- For clinical datasets or third party data, please ensure that the statement adheres to our policy

The authors declare that the data supporting the findings of this study are available within the paper and it's supplementary information files. The datasets generated during and/or analyzed during the current study are available from the corresponding author on reasonable request. 
Please select the one below that is the best fit for your research. If you are not sure, read the appropriate sections before making your selection.

\ Life sciences

Behavioural \& social sciences

Ecological, evolutionary \& environmental sciences

For a reference copy of the document with all sections, see nature.com/documents/nr-reporting-summary-flat.pdf

\section{Life sciences study design}

All studies must disclose on these points even when the disclosure is negative.

Sample size Sample size for animal experiments was determined based on criteria set by institutional ACUC. SARS-CoV- 2 is a novel virus and animal challenge models are still being optimized. There is not sufficient data available for the rhesus macaque challenge model to power an experiment to show a statistical difference between vaccine doses and that is not our goal. In this study, we aim to (1) assess immunogenicity with different doses and vaccine regimens (2) determine the durability of immunity and protection following vaccination. We are proposing to immunize eight animals at each vaccine dose. We anticipate reduced viral titers, clinical and radiographic signs and reduced histopathological changes in the lungs of the vaccinated animals. Eight animals is sufficient to provide $>80 \%$ power to detect significant protection as determined by the following endpoints: sgRNA and viral PCR in day 2 post-challenge BAL and NS. The information garnered by this experiment will inform subsequent experiments aimed at identifying a serological correlate of protection from SARS-CoV-2 disease.

Data exclusions No data were excluded.

Replication Animal studies were completed once as these are NHP studies. All immunoassay testing was completed in technical duplicate or triplicate with 1 replicate, unless otherwise stated in methods, and all replicates were successful.

Randomization Allocation of animals was randomized based on age and weight into groups on 8.

Blinding Blinding was not completed as assays were completed by the same team that immunized animals.

\section{Reporting for specific materials, systems and methods}

We require information from authors about some types of materials, experimental systems and methods used in many studies. Here, indicate whether each material, system or method listed is relevant to your study. If you are not sure if a list item applies to your research, read the appropriate section before selecting a response.

\begin{tabular}{l|l} 
Materials \& experimental systems \\
\hline $\mathrm{n} / \mathrm{a}$ & Involved in the study \\
\hline & $\bigotimes$ Antibodies \\
$\square$ & $\square$ Eukaryotic cell lines \\
$\square$ & $\square$ Animals and other organisms \\
$\square$ & $\square$ Clinical data \\
$\square$ & $\square$ Dual use research of concern
\end{tabular}

\begin{tabular}{l|l} 
Methods \\
\hline n/a & Involved in the study \\
$\square$ & $\square$ ChIP-seq \\
$\square$ & $\square$ Flow cytometry \\
$\square$ & $\square$ MRI-based neuroimaging
\end{tabular}

Antibodies

Antibodies used
Validation - costimulatory antibodies anti-CD28 and anti-CD49d (clones CD28.2 and 9F10, BD Biosciences)
No validation.

\section{Eukaryotic cell lines}

Policy information about cell lines

Cell line source(s)

HEK293T/17 (ATCC \#CRL-11268), Vero E6 (ATCC) Vero-TMPRSS2 (made in-house - VRC), ACE-2-expressing 293T (ATCC) cells (provided by Michael Farzan, Scripps Research Institute)

Authentication

None of the cells lines were authenticated.

Mycoplasma contamination

All cell lines were tested negative for mycoplasma. 


\section{Animals and other organisms}

Policy information about studies involving animals; ARRIVE guidelines recommended for reporting animal research

$\begin{array}{ll}\text { Laboratory animals } & \begin{array}{l}\text { Hamsters: Male and female Golden Syrian hamsters (Envigo, 089), aged 8-9 weeks old. } \\ \text { NHP: Male and female, 3-12 year-old, Indian-origin rhesus macaques. }\end{array} \\ \text { Wild animals } & \text { The study did not use wild animals. } \\ \text { Field-collected samples } & \text { The study did not use field-collected samples. } \\ \text { Ethics oversight } & \begin{array}{l}\text { Animal experiments were carried out in compliance with US National Institutes of Health regulations and approval from the Animal } \\ \text { Care and Use Committee of the Vaccine Research Center and Bioqual, Inc. }\end{array}\end{array}$

Note that full information on the approval of the study protocol must also be provided in the manuscript.

\section{Flow Cytometry}

Plots

Confirm that:

\The axis labels state the marker and fluorochrome used (e.g. CD4-FITC).

\The axis scales are clearly visible. Include numbers along axes only for bottom left plot of group (a 'group' is an analysis of identical markers).

\All plots are contour plots with outliers or pseudocolor plots.

$\bigotimes$ A numerical value for number of cells or percentage (with statistics) is provided.

\section{Methodology}

Sample preparation

Instrument

Software

Cell population abundance

Gating strategy
Rhesus macaque PBMC were isolated from whole blood using a Ficoll gradient and cryopreserved.

\section{BD FACSymphony A5}

Diva software was used to collect the data and FlowJo was used to analyze the data.

N/A as cells were not sorted.

T cell populations were selected by subsequent gating: first single cells, followed by live/SSC low cells, then CD3+/FSC low cells, and finally CD4+ and CD8+T cells. To measure IL-21 production and CD40L expression from Tfh, CD4 T cells were gated first on central memory (CM) T cells (CCR7+CD45RA-) then CXCR5+CM cells followed by PD-1+ICOS+ cells to identify Tfh. Subsequently, from Tfh, CD69+IL-21+ and CD69+CD154+ cells were gated. Cytokine production from total memory (TotM) CD4 and CD8 T cells was measured by first gating on total memory cells (central memory CCR7+CD45RA- plus effector memory CCR7-CD45RA- plus terminal effector memory CCR7-CD45RA+ T cells) while excluding naïve CCR7+CD45RA+ T cells, then CD69+cytokine+ gates were drawn on total memory T cells.

$\bigotimes$ Tick this box to confirm that a figure exemplifying the gating strategy is provided in the Supplementary Information. 